\title{
VIII. Von Kollektivierung zu Kollektivierung: Die Jahre 1954 bis 1958
}

\section{Die abgebremste Kollektivierung 1954 bis 1956}

\section{Agrarwirtschaftliche und-politische Rabmenbedingungen}

Die Agrarpolitik der SED in den beiden letzten Jahren des ersten Fünfjahrplanes 1954 und 1955 zielte darauf, die gesamtwirtschaftliche Versorgungslage zu verbessern ${ }^{1}$. Daher betrieb man eine doppelgleisige Politik, die aus ernährungswirtschaftlichen Motiven auf den Erfolg der privatwirtschaftlichen Agrarproduktion setzte und zugleich den Ausbau des genossenschaftlichen Sektors vorantrieb. Wegen der anhaltenden Mißwirtschaft des überwiegenden Teils der LPG war man davon abhängig, daß zumindest die private Landwirtschaft die Versorgung stabilisierte. Da die staatlichen Unterstützungsleistungen für die LPG den Fiskus zunehmend belasteten, konnte sich die SED ohnehin nur eine gewisse Quote an Kollektivwirtschaften leisten ${ }^{2}$. Gerade unter volkswirtschaftlichen Gesichtspunkten - Getreide- und Lebensmittelimporte strapazierten die Devisenbilanz - versuchte die Regierung, die Selbstversorgung mit Agrarprodukten zu erreichen ${ }^{3}$; außerdem besänftigte dieses Vorgehen die Stimmung der Bevölkerung.

Die Einstellung der Bauern seit 1952 hatte gezeigt, daß die wirtschaftlich Erfolgreichen nicht freiwillig in LPG eintraten, wenn diese sich überwiegend aus Landarbeitern und Neubauern auf unrentablen Höfen zusammensetzten ${ }^{4}$. Neben der politisch motivierten Abneigung gegen die LPG an sich und gegen die Partei, die dort dominierte, schreckte betriebswirtschaftlich denkende Bauern vom Eintritt auch die Praxis ab, derzufolge die Räte der Kreise den LPG devastierte Flächen zuweisen konnten, was deren Wirtschaftlichkeit unberechenbar machte.

Vor diesem Hintergrund hatte die Staatliche Plankommission 1953 empfohlen, den wirtschaftlichen Freiraum der Privatbauern zu vergrößern. Die Pflichtablieferungsnormen blieben seit 1953 konstant. Aufgrund veränderter Aufkaufpreise erzielten "großbäuerliche“ Produzenten Einnahmeverbesserungen, bisher geltende Benachteiligungen waren reduziert worden. Seit August 1956 galt für „Großbau-

1 Aus sowjetischer Sicht Berichte des Hohen Kommissars, z. B. Über Lage der DDR im ersten Halbjahr 1954, Foitzik, Berichte, hier S. 1440-1446. Zur Lage der Landwirtschaft siehe Noziczka, Politik, S. 8-37.

2 Die Subventionen mußten den garantierten Mindestsatz von 7 DM pro Arbeitseinheit der Mitglieder und die enormen Aufwendungen zur Ausstattung der LPG finanzieren.

3 Schulz, Probleme, S. 12; es ist nicht einsichtig, weshalb er andernorts den Pflichtablieferungszwang als "Abnahmegarantie“ interpretiert, die noch dazu als "soziale Sicherung“ akzeptiert worden sei. Schulz, Ruhe, S. 104.

4 Im Oktober 1953 zählten die LPG insgesamt 59900 Bauernwirtschaften mit 136300 Mitgliedern, darunter 35,4\% ehemalige Landarbeiter, 44,5\% Neubauern, 16,3\% Altbauern und $3,8 \%$ andere, Foitzik, Berichte, S. 1388. 
ern" bei MTS-Arbeiten der günstigere Mittelbauerntarif5. Der Viehhalteplan für 1956 wurde mit der Aussicht abgeschafft, im gleichen Jahr auch auf den Anbauplan zu verzichten. Die DBD begrüßte beides. Minister Reichelt gab allerdings zu bedenken, daß man in den Dörfern nach der Aufhebung der Anbauplanung nun die Abschaffung der Ablieferungspläne fordere ${ }^{6}$; die gewährte betriebswirtschaftliche Flexibilität rief also weitergehende Wünsche hervor. 1957 wurden die Anbauplanung für Getreide und Kartoffeln aufgehoben und die Erfassungspreise für Rindfleisch erhöht. Von dieser Preisanhebung profitierte vor allem die private Landwirtschaft, die einen höheren Viehbesatz aufwies als die LPG. Die Ablieferungsnormen, das Prinzip des doppelten Preissystems für Pflichtsoll bzw. „freie Spitzen" und die Art der Besteuerung landwirtschaftlicher Betriebe blieben jedoch unverändert".

Die SED stellte in Aussicht, die Anbauplanung abzuschaffen, die Pflichtablieferung nicht nur nach Betriebsgrößengruppen, sondern an den natürlichen Bedingungen zu bemessen und die Besteuerung zu vereinfachen. Ungeachtet aller Unzulänglichkeiten in der Produktionsmittelversorgung für die private Landwirtschaft bewirkten diese Erleichterungen, daß sich ein Teil der einzelbäuerlichen Betriebe wirtschaftlich stabilisierte, daher noch weniger bereit war, freiwillig in ökonomisch labile LPG einzutreten. Der Trend hielt an, Höfe lieber aus wirtschaftlichen Gründen ganz aufzugeben als sie in LPG einzubringen. Diejenigen Betriebe, die bis 1957 Genossenschaften beitraten, machten im Vergleich zur Zahl aller aufgegebenen nur ein Drittel aus ${ }^{8}$. Auch unsichere private Zukunftsperspektiven, etwa infolge von Krankheit, Tod und der Distanz der nachwachsenden Generation gegenüber dem landwirtschaftlichen Beruf, erhöhten die Quote an Betriebsaufgaben ${ }^{9}$. Angesichts der eigenen Bedrängnis im Kontrast zu den Angeboten der Industrie, die seit der 3. Parteikonferenz der SED die 45-Stunden Woche und den Sieben-Stunden-Tag avisierte, war die Jugend immer weniger bereit, den elterlichen Hof zu übernehmen oder mit ihm in die LPG einzutreten. Letzteres hätte die SED bevorzugt.

Seit dem Februar 1954 stieg die Zahl der LPG wieder an, doch dominierten unter den Neugründungen bis einschließlich 1955 solche aus ÖLB-Ländereien; nur rund ein Zehntel basierte auf dem Zusammenschluß von Bauernwirtschaften und Landarbeitern ${ }^{10}$. Daher wurden überwiegend LPG des Typs III gegründet, in denen man Acker- und Grünland gemeinsam bewirtschaftete und - falls vorhanden

5 Schulz, „Kapitalistische Länder überflügeln“, S. 8 f.; Nehrig/Noziczka, Weiterentwicklung, S. 1086.

6 ACDP VI-052-0315, Protokolle Präsidium, 1. 11. und 15. 11. 1955.

7 Nehrig/Noziczka, Weiterentwicklung, S. 1091; allerdings sollten die bäuerlichen Betriebe durch die Führung sogenannter Produktionsnachweise kontrolliert werden, in denen sie über Anbau, Viehbestand und die geplanten Erträge berichteten und deren Ergebnisse die $\mathrm{VdgB}(\mathrm{BHG})$ überwachte. Reichelt, Blockflöte, S. 154.

8 Schulz, „Kapitalistische Länder überflügeln“, S. 8. Von Mitte 1954 bis Mitte 1955 wurden 9747 Betriebe aufgegeben, 1955/56 stieg die Zahl auf 14297.

$940 \%$ der den ÖLB zugeführten Betriebe wurden wegen Krankheit und Tod aufgegeben; BA DK-1/460, Bl. 20-25, Bericht über die Bewirtschaftung freier Flächen und die Situation der ÖLB.

10 Schulz, „Kapitalistische Länder überflügeln“, S. 85, 88 . 
- das Vieh zusammenlegte. Dem stagnierenden Zuwachs versuchte die SED durch flexiblere Bedingungen für die LPG-Gründung entgegenzuwirken. So öffnete die III. LPG-Konferenz im Dezember 1954 die LPG unter gewissen Einschränkungen auch für sogenannte „Großbauern“, von denen im Jahr 1955 immerhin 2347 eintraten ${ }^{11}$. Aufgrund ihrer wirtschaftlichen Kraft und beruflichen Erfahrung erlangten sie häufig die Meinungsführerschaft unter den Mitgliedern, wenngleich ihnen Leitungspositionen offiziell verschlossen blieben. Wie sehr sie drängten, sich als legale Genossenschaftsbauern politisch zu betätigen, beweist ihr Bemühen, in den VdgB-Leitungen wieder Fuß zu fassen ${ }^{12}$. Umgekehrt belegt die Abwehr der SED, wie brüchig die Konstruktion der Genossenschaftsbauern als einheitliche „Klasse“ war, und in welch hohem Maße bisherige Schichtunterschiede lediglich in die Genossenschaften verschoben wurden. Die IV. LPG-Konferenz 1955 erlaubte, was bisher strikt untersagt war: In Dörfern, wo bereits eine LPG des Typs III bestand, durfte nun eine zweite LPG vom Typ I gebildet werden. Damit wollte man jenen Bauern, die auf keinen Fall für Typ III mit seinem hohen Grad an Vergenossenschaftlichung zu gewinnen waren, eine Wirtschaftsform nahebringen, die es erlaubte, weitgehend an der bisherigen Lebensweise festzuhalten $^{13}$. Dies sprach auch DBD-Mitglieder an. Denn häufig schreckte sie die SEDDominanz in den LPG davor ab beizutreten; auch mittelbäuerlichen Betrieben, die oft in sozialer Distanz zu den Mitgliedern der bestehenden LPG Typ III verharrten ${ }^{14}$, versprach diese Möglichkeit, ihren bisherigen Einfluß zu wahren.

Die SED-Agrarpolitiker stellten sich auf ein langjähriges Nebeneinander von privater und genossenschaftlicher Produktion ein und stifteten ein wirtschaftliches Konkurrenzverhältnis. Nur der Nachweis, daß der sozialistische Teil der Agrarwirtschaft überlegen sei, würde folglich die Einzelbauern zum freiwilligen Beitritt bewegen. Die ideologische Begründung ließ diese Konkurrenz jedoch nur für den privaten Teil zu einer Gefahr werden. Denn je bessere Ergebnisse er erzielte, desto stärker machte er sich in dieser verqueren agrarmarxistischen Logik verdächtig, daß der Mißerfolg der LPG auf das Einwirken des „Klassengegners“ außerhalb und innerhalb der LPG zurückzuführen und daher mit politischen Mitteln zu bekämpfen sei.

Seit November 1956 propagierten alle wichtigen agrarpolitischen Akteure SED, Ministerium, VdgB(BHG) und teilweise widerwillig auch die DBD - die Zusammenschlüsse der Bauern in sogenannten „Ständigen Arbeitsgemeinschaften". Auf diese einfache Form der Kooperation unter den Landwirten zu setzen, war die defensive Reaktion in der DDR auf die agrarpolitische Debatte im Ostblock im Herst 1956, sie basierte offenbar auch auf sowjetischen Wünschen. Man räumte den Arbeitsgemeinschaften jetzt Kredite und eine bevorzugte Behandlung durch die MTS ein, nachdem Bauern, die eine Arbeitsgemeinschaft gründen woll-

11 Ders., Probleme, S. 153 f.; Reichelt, Blockflöte, S. 152.

12 ACDP VI-052-1355, Protokoll Sekretariat BV Schwerin, 13. 5. 1957. So stellte etwa die CDU im Kreis Hagenow 20 „Großbauern“, getarnt als Kandidaten der VdgB, für die Kommunalwahlen auf.

13 Herferth, Aufschwung, S. 214.

14 Vgl. Langenhan, „Halte dich fern von den Kommunisten“, S. 138. 
ten, 1956 oft in LPG gezwungen worden waren, auch weil die MTS ihren Vertragsverpflichtungen nicht nachgekommen waren ${ }^{15}$. Die Arbeitsgemeinschaften verbanden ideologische und wirtschaftliche Ziele, indem sie sowohl an die genossenschaftliche Existenzform ,in einem begrenzten Zeitraum “16 heranführen, als auch die neuerliche Strukturkrise in ländlichen Regionen auffangen sollten. So wurden die Einzelbauern angehalten, in Gemeinschaftsarbeit die verlassenen Flächen zu bestellen und den ÖLB als Arbeitskräfte zur Verfügung zu stehen. Die Mittelbauern lehnten diese Gemeinschaften, die sie als Vorstufe der LPG ansahen, schon Mitte $1954 \mathrm{ab}^{17}$. Auch die VdgB(BHG) konstatierte Ende 1955, daß diese Gemeinschaften sogar dort, wo sie als solche ausgewiesen seien, nicht im SEDSinne funktionierten, sondern nur eine Form der herkömmlichen Nachbarschaftshilfe seien ${ }^{18}$, sich also im Spektrum traditioneller Arbeitsweise bewegten; im übrigen würden sich die Kreisverbände durch die Meldung falscher Erfolgszahlen aus ihrer Verantwortung stehlen, die Bildung und Existenz solcher Gemeinschaften zu fördern. Die Einzelbauern stießen sich am patriarchalischen Erziehungsstil, der ihnen vermeintlich "fortgeschrittenere" Genossenschaftsbauern als Vorbild präsentierte. Ebenso war es illusorisch zu glauben, daß sie aufgrund der Gemeinschaftsarbeit zur gewünschten Einsicht gelangen würden, daß Maschinen notwendigerweise vom Staat bereitgestellt werden müßten, weshalb deren Abgabe an Privatpersonen zu reduzieren sei ${ }^{19}$. Ungeachtet der begrenzten Akzeptanz waren diese Gemeinschaftsformen dennoch Teil der Debatte über die Genossenschaften in den Dörfern. Immerhin existierten im Sommer 19579026 "Ständige Arbeitsgemeinschaften", in denen 36318 überwiegend klein- bis mittelbäuerliche Betriebe sich gegenseitig aushalfen ${ }^{20}$.

Ein Merkmal der SED-Agrarpolitik lag darin, daß ihr offizieller Stellenwert je nach politischer Lage beliebig verändert wurde. Wurde ihr nur eine geringe Bedeutung beigemessen, so verminderte dies den agitatorischen Elan der Apparate und Kader, auf eine Sozialisierung hinzuarbeiten. So gab Mückenberger der DBD zwar intern zu verstehen, daß es im zweiten Fünfjahrplan zentral um die Probleme der Landwirtschaft gehe, weil ihre Entwicklung eine eminente Anzeigefunktion für das Erreichen des Sozialismus habe. Hingegen unterstrich Ulbricht im Zuge der Erlangung der Souveränität 1955 auf dem 25. ZK-Plenum erneut den

15 International Department, Rolle 109 (RGANI, 5-26-427, Bl. 178-187), Puschkin an ZK der KPdSU, Bericht über Entwicklung einfachster Formen der Kooperativen in der Landwirtschaft der DDR (erstellt von Kulikow), 10. 10. 1956.

16 So unmißverständlich Nehrig/Noziczka, Weiterentwicklung, S. 1089.

17 „In solchen Dörfern, wo das erkannt ist, gibt es unter den Mittelbauern keine Gemeinschaften der gegenseitigen Hilfe," so Rose. ACDP VI-052-0527, Protokoll erweiterte Sekretariatssitzung, 5. 7. 1954.

18 SAPMO VdgB 121, Protokoll Sekretariat VdgB, 11.11. 1955.

19 Ebd., Anlage 2, ideolog.-polit. Plan über Bedeutung und Rolle der ständigen Arbeitsgemeinschaften.

20 Die Durchschnittsgröße der Arbeitsgemeinschaften betrug 33,5 ha LNF. Nur 792 Betriebe in den Arbeitsgemeinschaften hatten zwischen 5-20 ha, ein knappes Fünftel zwischen 0,5-5 ha, 1,5\% über 20 ha. Graffunder, Dialektik, S. 117; Droste, Rolle, S. 101. 
Ausbau der Schwerindustrie ${ }^{21}$. Wie die gesamte Politik, so fungierte auch die Landwirtschaft im zweiten Fünfjahrplan als Experimentierfeld dafür, die Bundesrepublik zu überholen ${ }^{22}$. Die DBD begrüßte und übernahm offiziell all diese Vorgaben der SED-Agrarpolitik ${ }^{23}$.

Eine Reihe von Strukturdefekten blieb bis 1956 und darüber hinaus für die Landwirtschaft der DDR charakteristisch. Zunächst spiegelte die hohe Zahl der aufgegebenen Betriebe in geringerem Maße die Staatsflucht als vielmehr die Landflucht wider. Da man die verlassenen Flächen, deren Umfang Ende 1953 auf 697980 ha geklettert war und damit $10 \%$ der LNF betrug ${ }^{24}$, vor allem aus arbeitsökonomischen und finanziellen Gründen nicht in großem Stil den labilen LPG zuschlagen konnte, hatte eine Verordnung vom 3. September 1953 die Einrichtung von sogenannten Örtlichen Landwirtschaftlichen Betrieben (ÖLB) geregelt. Sie waren vorläufiges Auffangbecken für verlassene Flächen, Drehscheibe des einst privaten Bodeneigentums, des kommunalen Grundbesitzes und des Nutzungseigentums aus dem Bodenreformfonds für eine künftige Zuweisung an den genossenschaftlichen oder staatlichen Teil der Landwirtschaft. Sie wurden von den Räten der Kreise als Lohnarbeitsbetriebe geführt und strapazierten die Staatskasse immens ${ }^{25}$. Anfang 1957 stammten schon $55 \%$ der von LPG bewirtschafteten Flächen aus ÖLB ${ }^{26}$. An der regionalen Verteilung der ÖLB ist abzulesen, welch hohen Anteil die durch die Bodenreform geprägten Gebiete hatten. Dort war es weder gelungen, die Höfe zu konsolidieren, noch die ehemaligen Neubauern bzw. deren Kinder davon zu überzeugen, den LPG beizutreten. Das Gros der LPG-Mitglieder bis Ende 1955 stellten Landarbeiter - 90\% der Beitritte in diesem Jahr machten zuvor in den ÖLB beschäftigte Landarbeiter aus ${ }^{27}$. Vor allem aber

21 ACDP VI-052-0526, Protokoll Sekretariat, 15.3. 1954, Anlage 2, zu Mückenberger; Weber, Geschichte, S. 267.

22 Siehe dazu grundsätzlich Schulz, „Kapitalistische Länder überflügeln“, bes. S. 7.

23 Goldenbaum begrüßte z. B. den Aufbau der Schwerindustrie, ACDP VI-052-0315, Protokoll Präsidium, 1. 11. 1955.

24 SAPMO NY 4090/363, Bl. 226, Scholz an Grotewohl, 24. 2. 1954.

25 VO über die Bewirtschaftung freier Betriebe und Flächen und die Schaffung von Betrieben der örtlichen Landwirtschaft, 3. 9. 1953, in: GBI. DDR 1953, I, S. 983; Tümmler, Agrarpolitik, S. 48-54; Schulz, Probleme, S. 138-142.

26 BA DK-1/5640, Dienstbesprechung, 9. 1. 1957; Berichte über Durchführung der IV. Konferenz der Vorsitzenden und Aktivisten der LPG. Zum 1.4. 1954 umfaßten die ÖLB 414331 ha. Bis zum 1. 4. 1955 hatten sie an LPG allein 204370 ha, die $81 \%$ der Flächenabnahme ausmachten, übergeben, der Rest dürfte an VEG gegangen sein. Ein weiteres Jahr später verwalteten die ÖLB nur noch 111500 ha - daneben gab es 25000 ha herrenloses Land -, bis zum 30. 6. 1957 stieg die Fläche der ÖLB wieder auf 140108 ha; BA DK-1/ 1216, Bl. 81-83, HA LPG, Haberland, an Sekretariat des Ministers Reichelt, 1. 4. 1955. Dem Bericht zufolge wuchs die Fläche der ÖLB vom 1. 4. 1954 bis 28. 2. 1955 erneut um 103032 ha. Zum 28. 2. 1955 verteilte sich der Flächenanteil an ÖLB nach Bezirken folgendermaßen: Neubrandenburg 86032 ha, ein Großteil durch das Verlassen von Neubauernbetrieben verursacht, im Bezirk Schwerin 38610 ha, Dresden dagegen nur 4242 ha; BA DK-1/460, Bl. 20-25, Bericht über Bewirtschaftung freier Flächen und ÖLB 1957.

27 In den 6047 am 25. 11. 1955 bestehenden LPG mit insgesamt 196946 Mitgliedern machten die Landarbeiter rund die Hälfte (96146) aus, Neubauern gab es 58755 und Altbauern nur 18847. Fast die Hälfte aller LPG-Mitglieder war weiblich (92 485), noch 1956 lag der Anteil der Genossenschaftsbäuerinnen bei $40 \%$. Zahlen zu 1955 bei Schulz, Probleme, Tabel- 
waren die LPG kein Anliegen der nachwachsenden Generation, 1955 zählten sie nur 13343 jugendliche Mitglieder. Allein im ersten Halbjahr 1956 trat fast ein Viertel von ihnen wieder aus, bis September nahm ihr Anteil um ein Prozent an der Gesamtmitgliedschaft ab und betrug nur noch 5,7\% oder 1276628; Mitte 1954 hatte er noch bei $7,3 \%$ gelegen ${ }^{29}$. Außerdem verstetigte sich der Trend, daß immer weniger Familienangehörige der eingetretenen Einzelbauern ebenfalls LPG-Mitglieder wurden ${ }^{30}$. Insgesamt litt der genossenschaftliche und der staatliche Teil der Landwirtschaft Mitte der fünfziger Jahre unter der hohen Mitgliederfluktuation und der mangelhaften Arbeitsorganisation ${ }^{31}$. In den LPG und MTS fehlten politisch geschulte Kader ebenso wie fachlich qualifiziertes Personal; die ansonsten fachlich besser ausgebildeten vormaligen Privatbauern besaßen hingegen kaum das für notwendig erachtete "sozialistische" Wissen zur adäquaten Leitung eines solchen Betriebes. Die schon 1953 in Gang gesetzte, 1954 proklamierte und im März 1955 forcierte Aktion „Industriearbeiter aufs Land“ brachte durch finanzielle Vergünstigungen zwar tatsächlich ehemalige Landbewohner dorthin zurück - bis Ende 1955 hatte man 31422 Arbeiter und Angestellte dafür gewinnen können -, doch blieben diese oftmals nur kurze Zeit ${ }^{32}$. Allein zwischen November 1955 und Ende September 1956 verließ ein knappes Drittel der ehemaligen Industriearbeiter und der Angehörigen sonstiger Berufe die LPG wieder; bis dahin waren rund 16\% der Landarbeiter, die im November 1955 LPG-Mitglieder waren, wieder ausgetreten ${ }^{33}$.

Nur schwer ließ sich ein entscheidendes Strukturproblem der frühen LPG, die unzureichende fachliche Qualifikation des Personals, ausräumen. Die geringen Ausbildungskapazitäten verzögerten die Behebung dieses Defizits. Die Kollekti-

len, S. 153, 161; International Department, Rolle 109 (RGANI, 5-26-427, hier Bl. 91), Gespräch Scholz mit Kulikow, Sekretär der sowjetischen Botschaft, 26. 3. 1956; zu 1956 Graffunder, Dialektik, S. 123.

28 SAPMO DY 30 IV 2/7/24, Bl. 23-38, Bericht über Entwicklung LPG im I. Halbjahr 1956, 18. 9.1956.

29 SAPMO DY 30 IV 2/7/27, Bl. 100-135, hier Bl. 135, Entwurf: Vorschlag zu Entwicklung der Produktion in LPG bis 1960, 14. 11. 1956.

30 Der Eintritt von "Großbauern" wirkte sich positiv auf den Arbeitskräftebesatz der LPG aus, weil sie viele Angehörige mitzogen. Mit Stand vom 30. 9. 1956 traten pro 100 ehemaliger Einzelbauern zugleich in die LPG noch ein: 70 Angehörige von Neubauern, 71 von Altbauern, 95 von "Großbauern“. Angehörige von "Großbauern“, die erstmals am 28. 2. 1955 registriert wurden, machten damals sogar 101 aus. Demgegenüber verfügte die private Landwirtschaft über einen höheren Arbeitskräftebesatz; auf 100 Einzelbauern kamen 160 mithelfende Angehörige. SAPMO DY 30 IV 2/7/27, Bl. 100-135, hier Bl. 103 recto, 135, Entwurf: Vorschlag zu Entwicklung der Produktion in LPG bis 1960, 14. 11. 1956.

311954 standen den 62662 LPG-Eintritten 32690 Austritte gegenüber. Die Austritte von Einzelbauern lagen fast so hoch wie die Eintritte. Schulz, Probleme, S. 121.

32 36\% der bis Ende 1954 auf dem Land tätigen Industriearbeiter verließen dieses bis August 1955 wieder. Schulz, Probleme, S. 119-121. Zur Aktion siehe BA DK-1/88, Mückenberger an Reichelt, 11. 3. 1955, Bl. 50f. Bauerkämper, Aufwertung, S. 260 f. Zur LPG in Merxleben siehe Schier, Alltagsleben, S. $138 \mathrm{f}$.

33 SAPMO DY 30 IV 2/7/27, Bl. 38 ff. Entwurf: Vorschlag zu Entwicklung der Produktion in LPG bis 1960, 31.10. 1956; der Bericht auch zum folgenden. Ebenso: International Department, Rolle 109 (RGANI, 5-26-427, Bl. 92-94), Gespräch Scholz mit Kulikow, Sekretär der sowjetischen Botschaft in Berlin, am 13. 3. 1956, 26. 3. 1956. 
vierung in den fünfziger Jahren bewirkte daher zunächst eine Entprofessionalisierung der Agrarwirtschaft. Vor allem in der SED fehlten fachlich wie politisch geschulte Kader in der Landwirtschaft. In diesem Kontext notierte sie gelegentlich voller Neid, daß die DBD im Unterschied zu ihr über jüngere, landwirtschaftlich ausgebildete Kräfte verfüge ${ }^{34}$. Der Kadermangel behinderte die vorgesehenen Kontrollen in der Agrarpolitik und den ideologischen Ausbau der Politabteilungen der MTS ${ }^{35}$. Daher bemühte sich die SED ab 1955 zunehmend darum, die eigenen Lücken mit Personal aus der DBD zu schließen, vor allem um die SED-Parteiorganisationen in MTS, LPG und VEG auffüllen zu können, die bis dahin nicht flächendeckend bestanden ${ }^{36}$. Im Oktober 1956 bemühte sich Minister Hans Reichelt, der damit einen Politbüro-Beschluß vom Februar aufgriff, um ein Austauschprogramm für technische landwirtschaftliche Kader mit der Sowjetunion ${ }^{37}$.

Die Strukturprobleme in der Landwirtschaft überforderten sowohl die zuständigen Verwaltungsstellen samt Kontrollwesen als auch den Landwirtschaftsapparat der SED. Die SED versuchte stets, die Verantwortung dafür dem von einem DBD-Mann geführten Ministerium zuzuschieben, und wollte damit über die eigene Inkompetenz, Konzeptionslosigkeit und über Reibungsverluste bei der Implementierung neuer Vorgaben hinwegtäuschen. Kontrollgruppen monierten einerseits die „überspitzte Zentralisation“ des MLF, wenn die SED-Weisungen auf unterer Ebene nicht durchzusetzen waren und keine Bewegung "von unten“ für den „Kampf um die Erfüllung der Beschlüsse der Partei und der Regierung“ in Gang zu setzen war ${ }^{38}$. Zugleich warf man dem Ministerium vor, die Räte der Bezirke und Kreise zu übergehen, indem es selbst in die Betriebsabläufe der MTS, LPG und VEG intervenierte und diese lenkte. Mit diesem Vorgehen hatte das MLF im Grunde jedoch die in den Jahren zuvor geäußerte Kritik der ZKSK aufgegriffen. So hatte sich Paul Scholz 1953 bei Grotewohl gegen „Diskriminierung und Einschüchterungsversuche“ gewehrt, wie sie die ZKSK bei Überprüfungen im Ministerium an den Tag gelegt hatte ${ }^{39}$. Mit dieser in der Tat etwas umständlichen Praxis, einzelne Betriebe mittels Brigaden anzuleiten, reagierte das Ministerium auch auf die 1955 vorgebrachte Kritik der SED. Laut dieser gingen Fehlent-

34 Dies galt für den Bezirk Suhl (25,9\% Jugendliche unter den DBD-Funktionären, nur $7,2 \%$ unter den SED-Funktionären); SAPMO DY 30 IV 2/15/11, Analyse über die DBD, BV Suhl, 16. 2. 1955.

35 SAPMO DY 30 IV 2/5/1666, Bl. 35, Ulbricht an Fritz Kleinert, 2. 7. 1955, Statistik zur Fluktuation in den Politabteilungen der MTS. SAPMO NY 4090/363, Bl. $307 \mathrm{ff}$., Bericht der Kontrollabt. beim Präsidium des MR, Gruppe Land-, Forst- und Wasserwirtschaft, 23. 8. 1954, hier Bl. $332 \mathrm{f}$.

36 Zur Zusammensetzung der Parteiorganisationen in den MTS, LPG, VEG 1953 und 1954 siehe SAPMO DY 30 IV 2/5/1666; zu den VEG siehe Nehrig, Leitungspersonal.

37 International Department, Rolle 109 (RGANI, 5-26-427, Bl. 192-196), Gespräch Kulikow, Sekretär der sowjetischen Botschaft in Berlin, mit Reichelt am 8. 10. 1956, 17. 10. 1956.

38 SAPMO NY 4090/364, Bl. 73-99, Bericht der ZKSK, Gruppe Land-, Forst-, Wasserwirtschaft, 11. 1. 1955.

39 BA DK-1/161, Bl. 281-283, Scholz an Grotewohl, 22. 12. 1953. 
wicklungen und Pannen im sozialistischen Segment der Landwirtschaft auf die Tätigkeit angeblicher „Saboteure“ und „Agenten“ im Ministerium zurück ${ }^{40}$. Der unmittelbare Einsatz vor Ort sollte dies ausschließen.

Während die genossenschaftlichen und volkseigenen Teile der Landwirtschaft unter enormen Kosten staatlich alimentiert wurden - für die VEG konsultierte man eigens sowjetische Berater im Ministerium ${ }^{41}$-, glich die Situation der Privatbetriebe jener vor Beginn der verschärften Großbauernpolitik im April 1952. Um die Stimmung der bäuerlichen Bevölkerung nicht unmittelbar vor der Volkskammerwahl im Oktober 1954 zu verschlechtern, beschleunigte man - volkswirtschaftlich widersinnig - die Erfassung der Produktion noch während der laufenden Ernte ${ }^{42}$. Der privatbäuerliche Bedarf an Betriebsmitteln wurde bei planwirtschaftlichen Perspektivrechnungen nicht gebührend berücksichtigt. Die Rückstände in der Viehwirtschaft, welche weiterhin eine Domäne der Privatbetriebe blieb, wurden dadurch mitverursacht. Dieses Manko tadelten ministerielle Kontrollgruppen, die z. B. als Folge fehlenden Futters und zu geringer Stallkapazitäten nur feststellen konnten, daß Einzel- und Genossenschaftsbauern dazu übergingen, weibliche, also zur Mast ungeeignete Kälber nicht großzuziehen, sondern umgehend zu schlachten ${ }^{43}$.

Ende 1955 gehörten rund 200000 oder $15,6 \%$ aller Bauern LPG an. Trotz aller Anstrengungen bewirtschafteten die LPG 1957 nur ein Viertel der landwirtschaftlichen Nutzfläche ${ }^{44}$. Am Ende des ersten Fünfjahrplans stand fest, daß die Entwicklung der Landwirtschaft im Vergleich zur Industrie zurückgeblieben war ${ }^{45}$. Abgesehen von einer ideologischen Zielrichtung verfügte die SED über kein tragfähiges agrarpolitisches Konzept. Ulbricht ging davon aus, die Agrarfrage lasse sich über den industriellen Aufbau lösen. Darin folgte er sowjetischen Sichtweisen, die die DDR an der Grenze der Weltblöcke mehr als "Schaufenster" der industriellen Überlegenheit des Sozialismus gegenüber dem Kapitalismus propagierten, denn als agrarpolitisches Experimentierfeld ${ }^{46}$.

40 Insgesamt ermittelte man im MLF Anfang 1955106 „verdächtige“ Personen, quer durch alle Parteien und parteilose; die Existenz von familiären Westkontakten oder die Mitgliedschaft in der "Jungen Gemeinde " genügte für diesen Verdacht; SAPMO DY 30 J IV 2/202/ 63, Bericht über das MLF, 20. 1. 1955.

41 SAPMO NY 4090/366, Bl. 63-65, Mückenberger an Grotewohl, 28. 9. 1954.

42 SAPMO NY 4090/363, Bl. 295-299, ZKSK, Kontrollgruppe Erfassung u. Aufkauf, Bericht der Überprüfung der Arbeit der staatl. Organe bei Vorbereitung und Durchführung der Erfassung pflanzlicher Erzeugnisse der Ernte 1954, 18. 8. 1954.

43 Ebd., Bl. $307 \mathrm{ff}$., Kontrollabt. beim Präsidium des MR, Gruppe Land-, Forst- und Wasserwirtschaft, 23. 8. 1954. Vgl. auch die Monita von Scholz gegenüber Kulikow, Sekretär der sowjetischen Botschaft, 26. 3. 1956, in: International Department, Rolle 109 (RGANI, 526-427, hier Bl. $93 \mathrm{f}$.).

44 Reichelt, Blockflöte, S. 153; Werkentin, Strafjustiz, S. 93.

45 SAPMO NY 4090/364, Bl. 73-99, Bericht der ZKSK, Gruppe Land-, Forst-, Wasserwirtschaft, 11.1. 1955.

$46 \mathrm{Vgl}$. Lemke, Berlinkrise, S. $50 \mathrm{ff}$. 


\section{Die DBD zwischen Stagnation und Stabilisierung}

Ein Großteil der Mitglieder und der Kreisfunktionäre, ja sogar ein beachtlicher Teil von Bezirksfunktionären der DBD war maßlos enttäuscht, als sie im „Neuen Kurs" lediglich eine vordergründig mit weicheren Methoden vorgehende Taktik erkennen mußten, mit der zugleich der Ausbau des Überwachungsnetzes einherging, aber keine agrarpolitische Wende. Dies belastete den organisatorischen Zustand der Partei bis 1955 erheblich ${ }^{47}$. Eingezwängt in die Mitgliederbeschränkung durch die SED, rang die Organisationsabteilung um eine Stabilisierung des Mitgliederstandes und agierte gegen die Lethargie auf allen Ebenen. Ein Drittel der Kreisvorstände arbeitete Anfang 1954 nicht, und eine Reihe von Bezirksvorstandssitzungen waren mangels Beteiligung beschlußunfähig ${ }^{48}$. Ortsfunktionäre verweigerten die politische Tätigkeit in der eigenen Gemeinde und wollten nur in benachbarten Gegenden auftreten, wo sie weniger bekannt waren ${ }^{49}$. Der Generalsekretär versuchte dem Schwund entgegenzuwirken, indem er Anfang 1955 eine großzügigere Toleranzmarge für die Aufnahme neuer Mitglieder ausgab ${ }^{50}$. Wer die „freie Wirtschaft" fordere, sei als Mitglied durchaus akzeptabel, müsse dann eben entsprechend „erzogen" werden. Wer wegen Wirtschaftsstrafvergehen belangt werde - diese Prozesse setzten schon vor 1955 wieder ein ${ }^{51}$-, war nicht mehr wie 1952/53 als „Saboteur" zu verdammen, sondern habe durchaus Platz in der DBD.

Das Sekretariat in Dresden wies die Parteigliederungen an, Austrittserklärungen mit ungenügenden Begründungen nicht anzuerkennen, ganz besonders wenn sie von Bauern stammten ${ }^{52}$. Abgesehen von der erhofften Erhöhung der Mitgliederzahl sollte dieses Verfahren vor allem die eigenen Parteigliederungen aktivieren und disziplinieren. Ganz unorthodoxe Methoden wählte in diesem Zusammenhang die DBD im Kreis Perleberg: Weil die Partei dort jedes neu beigetretene Mitglied mit zwei Zentnern Futtermittel begrüßt hatte, wurde sie nur noch die "Zwei-Zentner-Partei“ genannt ${ }^{53}$. Zwar stieß die Mißachtung von „Hinweisen“ des ZK-Sektors BO auf SED-Kritik, die die fehlende „kämpferische Auseinandersetzung "in der DBD bemängelte und eine „ideologische Erziehung" der Mitglieder anmahnte. Im Endeffekt vergrößerte die DBD durch ihre dem politischen Meinungsklima auf dem Land angepaßte Vorgehensweise jedoch den eigenen Wirkungsradius und damit auch die Anbindung der Parteibasis an das Regime ${ }^{54}$. Die Grundeinheiten ersannen eigene Wege zur Stabilisierung ihrer Gruppe, etwa in der "Großbauernfrage“. „Großbauern“, die ihre „Pflichten“ erfüllten, Abga-

47 Bezirksberichte beklagten die argumentative Notlage, den „Neuen Kurs“ erfolgreich an die Mitglieder zu „verkaufen“, obwohl die Einzelbauern erfolgreicher wirtschafteten als die LPG. ACDP VI, unverz. Best. BV Schwerin, Tätigkeitsbericht Jan. 1954.

48 ACDP VI-052-0526, Protokoll Sekretariat, 23. 3. 1954.

49 Ebd., Protokoll Arbeitssekretariat, 18. 1. 1954, Bericht der Abt. Org., 13. 1. 1954.

50 ACDP VI-052-0529, Protokoll Sekretariat, 5. 2. 1955.

51 Werkentin, Strafjustiz, S. 93.

52 ACDP VI-052-0955, Sekretariatsvorlage BV Dresden zu Org.-Bericht IV. Quartal 1953, 8. 1.1954.

53 SAPMO DY 30 IV 2/15/1685, Hausmitteilung, 18. 2. 1955.

54 SAPMO DY 30 IV 2/15/3, Bericht des Sektors BO zur DBD, Sommer 1954. 
ben zahlten und der Ablieferung nachkamen, waren im Grunde bis zur Vollkollektivierung als einfache Mitglieder geduldet.

Im Bezirk Schwerin ergaben Überprüfungen, daß Ortsgruppen die Existenz von "großbäuerlichen" Mitgliedern verleugneten und damit deren Integration deckten. Dort wo "Großbauern“ sogar in Parteileitungen saßen, mieden sie die Teilnahme an Kreisdelegiertentagungen. Sie wollten nicht außerhalb des lokalen Bereichs, indem sie toleriert waren, auffallen, da sie vor der Kritik übereifriger Kollegen dort nicht sicher waren ${ }^{55}$. Teilweise verzichteten sie freiwillig auf ihre Wiederwahl, kandidierten aber statt dessen, wie in Rudolstadt, für die Gremien der VdgB ${ }^{56}$. Im Bezirk Dresden kürten die überwiegend „großbäuerlich“ geprägten Ortsgruppen wie in Ebenheit gezielt politisch unverdächtig scheinende Kollegen zu Vorsitzenden, deren Hofgröße knapp unter der kritischen Grenze von 20 ha lag57. In Dresden sah sich die DBD aber auch veranlaßt, den "großbäuerlichen“ Einfluß zurückzudrängen. Sie nominierte in mühsamen Diskussionen „Gegenvorstände“, riskierte jedoch bei „administrativer Entfernung “ der „Großbauern“, daß sich die gesamte Ortsgruppe dann dem Einfluß der Parteileitung entzog 58 .

Gab die DBD für 1954 - „das Jahr der großen Initiative“ - die Losung aus, in erster Linie die Existenz der bestehenden LPG zu gewährleisten und jene Einzelbauern, die sich völlig zurückgezogen hatten, wiederzugewinnen ${ }^{59}$, so strebte sie 1955 erneut die Gründung von LPG an. Daneben waren die Veränderungen in der internationalen politischen Konstellation auch in den erweiterten deutschlandpolitischen Aufgaben der DBD erkennbar. Seit der sowjetischen Zwei-StaatenDoktrin fiel 1954/55 die Tribüne des deutsch-deutschen Verhältnisses und damit die Aufgaben in der Deutschlandpolitik mehr und mehr der DDR zu60. Bei den Parteimitgliedern prallte die gesamtdeutsche Agitation weitgehend ab. Die Partei konnte sie in dieser Phase nur über agrarbezogene, vor allem agrarwirtschaftliche Themen erreichen. Das bedeutete umgekehrt aber nicht, daß die deutsche Frage aus dem Blickfeld der Basis verschwunden gewesen wäre; ein heftiges Aufflammen des Vereinigungswillens ohne sozialistische Vorzeichen ist im Jahr 1957 - Adenauer gewann erneut die Bundestagswahl - unter der DBD-Mitgliedschaft spürbar.

In die Konjunkturen der Deutschlandpolitik fügten sich auch die agrarpolitischen Aktionen in der DDR ein. Die schon vorher geäußerten Absichten zur neuerlichen Ausdehnung des genossenschaftlichen Bereiches und zur Errichtung der Großraumwirtschaft wurden erst Ende 1954 wieder in politische Aktion umgesetzt und auch die DBD darauf verpflichtet. Im Frühjahr 1954 empfanden es

55 ACDP VI, unverz. Best. BV Schwerin, Tätigkeitsbericht Jan. 1954.

56 ACDP VI-052-0526, Auswertung von Bezirksvorstandssitzungen im Sekretariat, 4. 2. 1954.

57 ACDP VI-052-0955, Protokoll Sekretariat BV Dresden, 11. 1. 1954.

58 Ebd., Protokolle Sekretariatssitzungen BV Dresden, 1. 2., 1. 3. 1954.

59 Dazu z. B. ACDP VI-052-0526, Protokoll Arbeitssekretariat, 18. 1. 1954, Plan zur Auswertung der Beschlüsse des 17. ZK-Plenums; ACDP VI-052-0955, Protokoll Sekretariat BV Dresden, 11. 1. 1954; ACDP VI-052-224/2, Arbeit der Agrarpol. Kommission im BV Cottbus, Arbeitsplan I. Quartal 1954.

60 Vgl. Weber, Geschichte, S. 255-259; Lemke, Berlinkrise, S. 26ff.; Amos, Westpolitik, S. 191-205. 
Mückenberger und die ZK-Abteilung Landwirtschaft ungeachtet der deklamatorischen Beibehaltung des Kollektivierungskurses noch als Defizit, daß die SED im Grunde keinen Kontakt zu den Einzelbauern hatte. Auch die Grundeinheiten bemühten sich nicht um einen solchen, vermieden vielmehr in "sektiererischer“ Arroganz eine tatsächliche Auseinandersetzung mit diesen ${ }^{61}$. Mückenberger hatte auch der DBD im März 1954 noch empfohlen, sich umsichtig an die Privatbauern heranzutasten, indem die Partei zwar der Bildung von LPG wieder mehr Aufmerksamkeit widmen solle, "man sich aber auch nicht überschlagen soll bei der Arbeit"62. Erst nach dem 21. Plenum des ZK der SED in November 1954 schärfte der Sektor BO der DBD wieder ein, daß sie sich der Ausdehnung der LPG-Gründungen, der Schaffung einer Großraumwirtschaft und dem sowjetischen Neuererwesen zuwenden müsse ${ }^{63}$. Im Glauben an die Wirkung von kulturell aufgeladener Propaganda empfahl der Sektor hierfür, die Mitglieder durch Vorträge, Zirkel und Filmvorführungen weiterzubilden. So hoffte die SED, die labilen LPG gerade durch den Beitritt von wirtschaftlich erfolgreichen und politisch vorgebildeten Mitgliedern der Bauernpartei zu sanieren. Das Sekretariat der DBD beschloß im März 1955, mittelbäuerliche Mitglieder für den Eintritt in jene LPG zu gewinnen, die aus ÖLB hervorgegangen waren und sich daher in einer besonders kritischen Lage befanden ${ }^{64}$.

Die Zielrichtung des Sektors BO ging 1955 über die reine Schadensbegrenzung, die während des „Neuen Kurses“ im Mittelpunkt gestanden hatte, hinaus. Ungeachtet aller strukturellen Probleme der genossenschaftlichen Wirtschaftsform forderte der Sektor bzw. die Arbeitsgruppe BO 1955 eine ideologische Offensive in der Parteitätigkeit der DBD. Anläßlich der Durchführung ihres IV. Parteitages 1955 wurde die programmatische Arbeit eingehend unter die Lupe genommen. Die Arbeitsgruppe kritisierte insbesondere die Parteitagsrede Goldenbaums, die sich in völlig unzureichender Weise den ideologischen Brennpunkten zugewandt habe. Vor allem die Frage des Bündnisses mit der SED sei ungenügend behandelt worden, obgleich in vorangegangenen Kreisdelegiertentagungen und Bezirksparteitagen alarmierende Mängel in dieser Frage unter den Mitgliedern offenkundig geworden waren ${ }^{65}$. Diese Kritik wurde auch für innerparteiliche Ränke genutzt. Goldenbaum hatte im Vorfeld versucht, den Generalsekretär aus den Beratungen über ein neues Parteiprogramm und die Bildung eines schlagkräftigeren exekutiven Parteigremiums, des Präsidiums, herauszuhalten - dies wäre de facto auf eine Entmachtung Roses hinausgelaufen ${ }^{66}$. Darauf konterte Rose und warf ihm vor, er

61 SAPMO NY 4090/363, Bl. 243 f., Mückenberger an Sekretäre für Landwirtschaft der BL und KL, 10.3. 1954.

62 ACDP VI-052-0526, Mückenberger in Sekretariatssitzung der DBD, 15. 3. 1954.

63 SAPMO DY 30 IV 2/15/4, Disposition zu Schlußwort des 21. Plenums, 9. 11. 1954. Ebd., Sektor BO, Auswertung des 21. Plenums mit Vorschlägen für die DBD, 27. 11. 1954.

64 ACDP VI-052-0529, Protokoll Arbeitssekretariat, 21.3. 1955.

65 SAPMO DY 30 IV 2/15/50, Einschätzung des 4. Parteitages durch die AG BO, 7. 9. 1955. ACDP VI-052-0529, Protokoll Arbeitssekretariat, 5. 2. 1955, bes. Wettbewerb zur Vorbereitung des 4 . Parteitages.

66 Siehe dazu ACDP VI-052-0530, Protokoll Arbeitssekretariat, 28. 6. 1955. Von dieser Sitzung ließ Rose auszugsweise Protokolle anfertigen, die der Kaderakte von Scholz beige- 
habe auf dem Parteitag eine "ungenügende politische Orientierung “ gegeben ${ }^{67}$. Von den ursprünglichen Plänen wurde schließlich nur die Neugliederung der Gremien realisiert, die Programmänderung erst nach sorgfältiger Vorbereitung auf dem nächsten Parteitag 1957 beschlossen. Durch das Sekretariat der DBD verlief spätestens seit 1955 ein tiefer Graben. Obgleich die SED die Auseinandersetzungen benutzte und teilweise auch schürte, war die DBD im wesentlichen doch Opfer ihrer hausgemachten Machtkämpfe, die eine karrierebeflissene Funktionärselite immer wieder anfachte.

Während sich die Spitze selbst schwächte, wurde das Verhältnis zur SED seit 1953 zunehmend an der Basis diskutiert, insbesondere dort, wo die DBD mehr Mitglieder als die SED zählte und aus eigener Sicht politisch wie fachlich einwandfrei in den LPG arbeitete. Warum die DBD nicht die führende Partei der Bauern sein könne, wenn die SED die Partei der Arbeiter sei, zog sich als Argument der Basis stereotyp durch die gesamten fünfziger Jahre ${ }^{68}$. Die SED fürchtete um eigene Positionen auf dem Land. Die DBD sollte daher strikt auf den ihr zugeteilen Aufgabenbereich beschränkt werden. Sie sollte Mitglieder gezielt dort werben, wo sie damit LDPD und CDU zurückdrängen konnte. Ihr Schulungsprogramm durfte sich nur mit politischen Themen an die eigenen Mitglieder richten; es war ihr strikt untersagt, mit fachlich ausgerichteten Vorträgen eine größere Zuhörerschaft anzusprechen, was viel aussichtsreicher für die Werbung von Mitgliedern gewesen wäre ${ }^{69}$. Während eine Zusammenarbeit mit der SED auf unterer Ebene bei ideologischen Fragen brenzlig war - weil man unweigerlich den sensiblen Punkt der „Bündnisfrage“ berührte -, gelang der Kontakt in fachlichen Angelegenheiten besser ${ }^{70}$.

In zwei Punkten unterschied sich die DBD 1955 wesentlich von der SED. Erstens verfügte die Bauernpartei über einen besseren Zugriff auf die Einzelbauern. Dies war allein schon aufgrund ihrer Mitgliederstruktur vorgegeben, in der Einzelbauern insgesamt, aber auch in den Leitungen nach wie vor dominierten ${ }^{71}$. Sogar der Leiter der ZK-Abteilung Landwirtschaft, Mellentin, stellte 1955 hilflos fest, die SED „beschäftigt sich nur mit dem fortschrittlichsten Teil [...] aber mit der Masse der Landbevölkerung beschäftigt sich keiner"72. Zum zweiten zeichnete sich die DBD durch eine vergleichsweise realistische Einschätzung der agrarischen Situation aus, während MfS und SED in die ideologische Offensive zogen und Fehlentwicklungen nur als „Sabotage“ erklären konnten, damit aber die tat-

fügt wurden und offenbar dazu beitrugen, daß dieser von Kontakten zur SED ausgeschlossen wurde. Die Existenz dieser Dokumente wurde erst 1960 im Sekretariat bekannt und trug zur Ablösung Roses bei; siehe ACDP VI-052-0543, Protokolle Sekretariatssitzungen, 16. 1. 1960, 6. 2. 1961, 27. 2. 1961; ACDP VI-052-0544, Protokoll Sekretariat, 29. 3. 1961; siehe Kap. IX.6, S. 469, 522.

67 ACDP VI-052-0315, Protokoll Präsidium, 3. 11. 1955.

68 Z. B. SAPMO DY 30 IV 2/15/18, Bericht BL Halle an Sektor BO, 4. 3. 1957.

69 SAPMO DY 30 IV 2/15/4, Sektor BO, Disposition für Schlußwort, 9. 11. 1954.

70 SAPMO DY 30 IV 2/15/14, Sektor BO an Mückenberger, 17. 12. 1954.

71 Siehe Tabellen 6 und 19 und ACDP VI-052-154/1, Teil 2, Aufstellung über Anteil der EB und LPG-Mitglieder an Gesamtmitgliedschaft zum 20. 8. 1955 und über deren Anteile in den OG-Vorständen zum 27. 4. 1955, 16. 12. 1955.

72 SAPMO DY 30 IV 2/7/75, Sitzungsprotokoll ZK-Abt. LW, 24. 6. 1955. 
sächlichen Ursachen nicht erfaßten. Erst allmählich stellte die SED in langwierigen Untersuchungen fest, daß weniger der „Klassengegner“ als vielmehr Straftaten und Fehlverhalten in den sozialistischen Unternehmen die Mißwirtschaft dort begünstigten ${ }^{73}$. Einen erfrischend aussagekräftigen Gehalt weisen in diesem $\mathrm{Zu}$ sammenhang die ersten Protokolle des 1955 neu gebildeten Präsidiums auf. Dort konstatierte man eine schleichende Krise der Agrarwirtschaft und wandte sich vor allem Problemen zu, die in der Umsetzung der agrarpolitischen Maßnahmen auftraten. Die Bezirkssekretariate würden „einige Fragen der MTS sehr kritisch“ einschätzen. „Stark kritisiert wurde die Frage des Instrukteurunwesens. Es wurde gesagt, daß die Instrukteure in großer Zahl in die Dörfer kommen, aber praktisch nichts verändern. “74 Über die Stimmung in der ländlichen Bevölkerung gab man sich keinen Illusionen hin. Rose konstatierte, daß „das Dorf“ sich zwar mehr für politische Themen interessiere als vordem, aber deshalb den Regierungsbestimmungen gegenüber nicht aufgeschlossener sei, vielmehr habe "die Feindtätigkeit“ wieder zugenommen ${ }^{75}$. Um eine Wende in der politischen Tätigkeit mit der Basis herbeizuführen, plädierte Hans Schnitzler für eine Neubesinnung auf die dortige Stimmung, „um diese Meinung mit den grundsätzlichen Problemen zu verbinden. Es muß daher mit den fertig ausgearbeiteten Rededispositionen Schluß gemacht werden. Es soll mehr eine Anleitung sein, um darum in Referaten usw. die örtlichen Bedingungen berücksichtigen zu können." Die Popularisierungsstrategien des MLF hielt er für unzureichend. Die Argumentationen, die es begleitend zu den Maßnahmen herausgebe, entsprächen in keiner Weise den Tatsachen und seien „direkt unehrlich“. Notwendig sei hingegen, eine „offene, ehrliche politische Argumentation herauszugeben " 76 .

Diese Elemente der Neuorientierung wurden vom Funktionärsapparat der DBD-Zentrale keineswegs dazu genutzt, um genuine Interessen der bäuerlichen Bevölkerung aufzugreifen, die mit dem herrschenden agrarpolitischen Kurs unvereinbar waren. Bäuerliche Interessen brachte die DBD mit einigem Erfolg nur dann ins Spiel, wenn es darum ging, Reibungswiderstände zwischen dem agrarpolitischen Kurs der SED und den realen Verhältnissen zu mindern und damit das Regime zu stabilisieren.

73 Siehe z. B. BStU MfS unsign. Akt, Abt. Wirtschaft, Dienstanweisung Nr. 38/55, 14. 10. 1955. Nr. 002113, 8 Bl., Bearbeitung von Havarien, Katastrophen und Bränden in Industrie und Landwirtschaft. Vgl. Lindenberger, ABV, S. 174-176.

74 ACDP VI-052-0315, Protokoll Präsidium, 28. 9. 1955. Goldenbaum fehlte auf dieser Sitzung wegen Urlaub.

75 Rose hielt die Behauptung für falsch, daß sich DDR-Bauern anläßlich Adenauers Moskau-Besuch „darüber beschwert haben, daß Adenauer nicht die Helden geehrt hat, sondern die Kirche besuchte. Den Bauern liegt die Kirche näher als die Helden der Sowjetunion. Wir dürfen uns deshalb keinen falschen Illusionen hingeben." Ebd.

76 Ebd., Protokoll Präsidium, 1. 11. 1955. 


\section{Agrarpolitik in der Diskussion 1956/1957}

\section{Die agrarpolitische Konzeption von Kurt Vieweg und Marga Langendorf und die politische Reformdiskussion 1956}

Auf dem XX. Parteitag der KPdSU im Februar 1956 vollzog die sowjetische Führung einen Bruch mit Stalin und Teilen seiner Methoden und vertauschte einige ältere ideologische Erklärungsmuster mit neuen Konzepten ${ }^{77}$. Die Stalinkritik erreichte zwar die zum Kongreß angereisten Parteiführungszirkel der Ostblockstaaten und drang auch in westliche Medien vor, öffentlich wurde die Bevölkerung im kommunistischen Machtbereich jedoch erst 1961 von Stalins Verbrechen und der Neubewertung seiner Rolle informiert. Die KPdSU propagierte eine „friedliche Koexistenz" der verschiedenen Gesellschaftssysteme. Es waren wieder unterschiedliche Wege zum Aufbau des Sozialismus denkbar, angeblich sogar ein parlamentarischer. Die beiden letzten Punkte berührten die deutsche Frage, insofern als Alternativen zur Deutschlandpolitik der DDR, besonders bezüglich der Rolle des Parteienblockes, möglich schienen.

Stärker als in der DDR wirkten die Ergebnisse des XX. Parteitages in Ungarn und Polen auf laufende Reformdiskussionen ein. In agrarpolitischer Hinsicht ist bedeutsam, daß am kollektivwirtschaftlichen Kurs erheblich gerüttelt wurde. Vor allem das polnische Beispiel lieferte dafür wichtige Anstöße. Der wiedereingesetzte Gomulka, der 1948/49 auch aufgrund seiner Vorbehalte gegenüber Kollektivwirtschaften abgelöst worden war, beendete die einseitige Förderung der Produktionsgenossenschaften. Als Folge dieses Politikwechsels löste man unrentable LPG auf; ihre Zahl schrumpfte bis Ende 1956 auf ein Fünftel und lag bei nur noch 170078 .

Die Übernahme der neuen sowjetischen Flexibilität prallte in der DDR bekanntlich an Ulbrichts hartem Kurs gegen politisch Andersdenkende und innerparteiliche Konkurrenten ab ${ }^{79}$. Die 3. Parteikonferenz (24. bis 30. März 1956) ging im offiziellen Teil eher ausweichend auf die Frage ein, wie Stalins Politik in der DDR aufgenommen worden war und welche Verantwortung dafür Ulbricht, stellvertretend für die SED, trug8 ${ }^{80}$. Im Zentrum der Konferenz standen wirtschaftliche Themen. Die SED beschloß eine Direktive zum zweiten Fünfjahrplan, die als Kernelement Modernisierung durch „wissenschaftlich-technische Revolution" vorsah; sie versprach unter anderem, die industrielle Bruttoproduktion bis 1960 um „wenigstens 155 Prozent des Standes von 1955“81 und das Volkseinkommen um $45 \%$ zu steigern. Mit einer flexiblen Politik hoffte man die Neugründung von LPG voranzubringen, indem man mehr als bisher das Potential zur Genossenschaftsbildung unterhalb der LPG Typ III ausschöpfen wollte und infolgedessen auf Typ I und seine Attraktivität für Mittelbauern zählte. Allerdings setzte

77 Zum folgenden siehe Staritz, Geschichte, S. 144-150; Weber, Geschichte, S. 275-283.

78 Quaisser, Ende, S. 41 ff.; Fejtö, Geschichte, Bd. 2/2, S. 134.

79 Siehe dazu Klein, Parteisäuberungen, S. 46-65.

80 Weber, Geschichte, S. 279f. Zur Rezeption in einer Geheimsitzung der SED siehe Grieder, Sicht, S. 565.

81 Protokoll der 3. Parteikonferenz der SED, S. 62. 
man dies zumindest laut sowjetischen Einschätzungen bis Herbst 1956 nicht hinreichend $\mathrm{um}^{82}$.

Dennoch gaben die sowjetischen Ereignisse auch in der DDR einer Reformdebatte Auftrieb, die vornehmlich von Intellektuellen getragen wurde. Wie schon in der Krise 1953 konnte der Erste Sekretär auch dieses Mal die Diskussion letztlich dafür nutzen, um Parteikonkurrenten als „Opponenten“ zu brandmarken und als

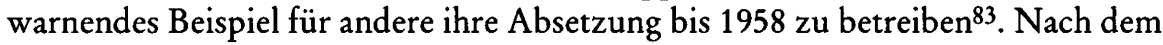
XX. Parteitag verdichtete sich in verschiedenen Institutionen und Gruppen, darunter auch im Führungszirkel der SED, die agrarpolitische Debatte, die offener als vorher ihre bisherigen Grundlagen in Frage stellte. Als bekanntestes Beispiel dafür gilt gemeinhin der Fall von Kurt Vieweg. Die an der Ausarbeitung des „Agrarprogrammes" beteiligte Assistentin Viewegs, die Wirtschaftswissenschaftlerin Marga Langendorf, steht in den einschlägigen Darstellungen im Schatten des einstigen Sekretärs für Landwirtschaft des $\mathrm{ZK}^{84}$. Die Kriminalisierung der beiden wurde dadurch erleichtert, wenn nicht sogar erst herbeigeführt, daß sie, verängstigt durch die öffentliche Kritik an ihrem Programm auf dem 30. Plenum des ZK, eine Verhaftung fürchteten und in die Bundesrepublik flohen; sie kehrten im Herbst 1957 in die DDR zurück und wurden 1959 verurteilt, Vieweg zu zwölf Jahren Haft, Langendorf zu sieben ${ }^{85}$.

Bislang blieb unberücksichtigt, daß Vieweg sich offensichtlich bis unmittelbar vor dem 30. ZK-Plenum am 30. Januar 1957 in Sicherheit wiegte und sogar noch Forderungen bezüglich seiner künftigen Tätigkeit in der DAL bzw. seiner und Langendorfs Position dort stellte ${ }^{86}$. Die Angelegenheit erhielt durch zwei Faktoren eine eigene Dynamik: Zum einen hatten sich beide in der DAL persönliche Feinde gemacht; der andere, gewichtigere Punkt war jedoch, daß Ulbricht den agrarpolitischen Kurs in der DDR ganz eng mit der Frage des Sozialismus überhaupt verknüpfte. Die Ereignisse in Polen und Ungarn im Herbst 1956, die dort zu einer Auflösung von LPG geführt hatten, beeinflußten auch die Behandlung des „Falls Vieweg“. Ulbricht warf diesem auf der 30. ZK-Tagung explizit vor, seine Vorschläge stünden in Verbindung mit der Modifizierung der Agrarpolitik in den „Bruderstaaten“. Auf der gleichen Veranstaltung berichtete Honecker über eine Politbürositzung, in der man den Besuch einer SED-Delegation bei der Pol-

82 International Department, Rolle 109 (RGANI, 5-26-427, Bl. 178-187), Puschkin an ZK der KPdSU, Bericht über Entwicklung einfachster Formen der Kooperativen in der Landwirtschaft der DDR (erstellt von Kulikow), 10. 10. 1956.

83 Staritz, Geschichte, S. 151-169; Weber, Geschichte, S. 283-296. Vgl. besonders Klein u. a., Visionen.

84 Vieles deutet darauf hin, daß sie in gleicher Weise wie Vieweg daran beteiligt war. Anders Scholz, Bauernopfer, der sich fast ganz auf Vieweg konzentriert. Die MfS-Überlieferung legt hingegen nahe, den Anteil Langendorfs, die der "theoretische Kopf“ des Teams gewesen sei, als bedeutsamer einzuschätzen.

85 Siehe dazu Scholz, Bauernopfer, S. 188-201, 207 f.; Beckert, Instanz, S. 212-218.

86 SAPMO DY 30 IV 2/7/561, Bl. 89-107, Protokoll Mitgliederversammlung der DAL, am 28/29. 1. 1957. Vieweg unterstrich, er werde die Akademie verlassen, falls man Langendorf kündige; Bl. 117, Kurzprotokoll der Versammlung, angefertigt von Gen. Heinrich, demnach sei Vieweg bereit, Langendorf zu entlassen, ohne selbst als Direktor zurückzutreten. Erst nach Eröffnung des Parteiverfahrens reichte er sein Rücktrittsgesuch ein. 
nischen Vereinigten Arbeiterpartei im Dezember 1956 in Polen besprochen hatte. Bei dieser deutsch-polnischen Zusammenkunft sei auch die Frage der LPG erörtert worden ${ }^{87}$.

Die Diskussionen blieben nicht auf Vieweg und die von ihm geleitete DAL beschränkt. Eine Krisenanalyse erstellte auch die ZK-Abteilung Landwirtschaft, die sich auf Materialien des MLF und der Staatlichen Plankommission stützte. Die Unzulänglichkeiten der Agrarstatistik erschwerten eine exakte Lagebeurteilung, denn die DDR verfügte bis dahin über kein solches, nach verläßlichen Kriterien erarbeitetes Zahlenwerk. Insbesondere im Ministerium, das Statistiken erarbeiten oder zumindest Daten beisteuern mußte, vertraten auch altgediente Genossen Auffassungen, die nach dem Bekanntwerden der Vorgänge in der DAL ebenfalls als „abweichlerisch“ gebrandmarkt wurden. Im November 1956 zog die ZK-Abteilung Landwirtschaft unter Federführung des neuen Leiters Franz Mellentin Bilanz über den Zustand der LPG. Völlig entgegen den vorgelegten Fakten des Ministeriums vertrat die Abteilung die Ansicht, man könne nicht folgern, „daß die LPG in ihrer Entwicklung nicht vorangekommen wären"88. Tatsächlich war die Zunahme des genossenschaftlichen Teils der Landwirtschaft gerade im dritten Quartal 1956 ganz klar rückläufig. Hierfür machte die Abteilung die Vernachlässigung der LPG Typ I und II durch den Wirtschafts- und Staatsapparat verantwortlich, die diese Formen aufgrund des niedrigeren Vergesellschaftungsgrades der Produktionsmittel für ideologisch minderwertiger als die des Typs III hielten und entsprechend behandelten. Da sie diesen Typen keine längerfristige Existenz zubilligten, unternahmen sie keine besonderen Anstrengungen, diesen betriebswirtschaftlich zu helfen ${ }^{89}$.

Die vorläufigen Ernteeinschätzungen durch die staatliche Zentralverwaltung für Statistik, die, wie die Abteilung mahnte, hinsichtlich der LPG tendenziell zu hoch liegen würden, ergaben eindeutig, daß deren Produktivität sogar in der pflanzlichen Produktion unter derjenigen der Privatbetriebe lag90. Das war alarmierend, denn aus betriebswirtschaftlicher Sicht verfügten die LPG aufgrund ihrer größeren Fläche bei entsprechender maschineller Ausstattung über günstigere Bedingungen im Pflanzenanbau als Einzelwirtschaften. Offenbar setzten sie diesen Vorteil jedoch nicht erfolgreich um. Daß die kleineren Einzelbetriebe bei der arbeitsintensiven und auf Erfahrung beruhenden tierischen Produktion weit bessere Ergebnisse als die LPG erzielten, mußte die SED ohnehin in Kauf nehmen ${ }^{91}$.

87 SAPMO DY 30 J IV 2/1/170, Protokoll 30. ZK-Tagung, 30.-1. 2. 1957, Bl. 10 (Honecker), Bl. 113; vgl. Beckert, Instanz, S. 112.

88 Das folgende nach: SAPMO DY 30 IV 2/7/27, Bl. 100-135, Entwurf: Vorschlag über die Entwicklung der Produktion in den LPG bis 1960, 14. 11. 1956.

${ }^{89}$ Ebd., Bl. 101. Im übrigen lagen sie damit ganz auf der Linie Ulbrichts, der selbstredend den Typ III gegenüber den anderen Typen positiv hervorhob. Siehe z. B. Protokoll des III. PT, Bd. 1, S. 117. Zur Analyse des Zustands der LPG 1956 vgl. auch BA DE-1/3190, Bl. 9-28, Auswertung der Jahresendabrechnung der LPG für 1956, 19. 3. 1957, Bl. 11, $27 \mathrm{f}$.

90 In der DDR-Literatur wurde gewöhnlich die wirtschaftliche Überlegenheit der genossenschaftlichen Produktionsform gegenüber den Einzelbetrieben anhand von Zahlen der pflanzlichen Produktion zu belegen versucht. Ungeachtet dieser, nicht als „revisionistisch“ abzutuenden Analyse des ZK, z. B. Nehrig/Noziczka, Weiterentwicklung, S. 1085.

91 Sogar die züchterisch wertvollen Herdbuchkühe in den LPG wiesen eine durchschnittlich 
Insofern blieben der SED keine ökonomischen Argumente für einen LPG-Eintritt, mit denen betriebswirtschaftlich rational vorgehende Bauern noch am ehesten zu gewinnen gewesen wären. Die SED wußte, daß ihre dennoch mit wirtschaftlichen Begründungen gespeisten Kampagnen auf tönernen Füßen standen. 1955 hatte knapp ein Viertel (23\%) aller LPG keine Einkünfte erzielt, fast ein Drittel $(31 \%)$ hätte ohne staatliche Unterstützung überhaupt keinen Arbeitslohn für die geleisteten Arbeitseinheiten auszahlen können. Nur jede zehnte LPG erwirtschaftete den vorgesehenen Betrag von 7 DM pro Arbeitseinheit. Auf DBDÜberprüfungen basierend, führte der Bericht an, daß zum 30. September 1956 $70 \%$ der LPG ihre Produktions- und Finanzpläne nicht erfüllen und somit auch die erhaltenen Kredite nicht begleichen könnten ${ }^{92}$.

Mit keiner Silbe führte der Bericht die völlig insuffizienten Leistungen der MTS an; in einem Seitenhieb erlaubte man sich jedoch darauf hinzuweisen, daß Mitarbeiter der MTS im Vergleich zu Angestellten der staatlichen oder kommunalen Verwaltung weitaus höhere Löhne erhielten ${ }^{93}$. Mit mehr als 100000 Beschäftigten und einem Organisationsgrad von rund 23\% SED-Mitgliedern waren die MTS eine wichtige Bastion der SED ${ }^{94}$, von der sie die DBD nicht ohne Grund fernhielt.

Über die strukturelle und ökonomische Krise debattierten Agrarfunktionäre an verschiedenen Institutionen, im Zentrum Berlin ebenso wie an der Peripherie. Fred Oelßner sprach sich auf der 28. und 29. ZK-Tagung im August und November 1956 für eine Auflösung der unrentablen LPG und MTS aus und regte an, das Tempo der Vergenossenschaftlichung zu drosseln ${ }^{95}$. Er stritt um diese Auffassungen im Dezember 1956 auch im Politbüro ${ }^{96}$. Oelßner ordnete die Probleme der

schlechtere Leistung auf jene Tiere der privaten Landwirtschaft, die für die Züchtung nicht anerkannt waren. SAPMO DY 30 IV 2/7/27, Entwurf: Vorschlag über die Entwicklung der Produktion in den LPG bis 1956, 14. 11. 1956, hier Bl. 105-107.

92 Ebd., Bl. 113 verso.

93 Das führte zu der Schieflage, derzufolge ein Kreisagronom, der die Oberagronomen in den MTS anleiten sollte, im Schnitt weniger verdiente (564 DM/Monat) als diese $(840$ $\mathrm{DM} /$ Monat). Im Vergleich zu letzteren verdiente ein Abteilungsleiter im DBD-PV nur unerheblich mehr (rund 900 DM). Ebd., Bl. 116. Die MTS waren unantastbar. Auch Nehrig/Noziczka versuchen den Eindruck zu erwecken, daß Probleme dort erst infolge des revisionistischen Auftretens Viewegs entstanden. Nehrig/Noziczka, Weiterentwicklung, S. $1093 \mathrm{f}$.

94 Ebd., Weiterentwicklung, S. 1083.

95 Oelßner war im PB und im Präsidium des MR für Versorgungsfragen zuständig und daher auch mit landw. Problemen befaßt. Schulz, „Kapitalistische Länder überflügeln“, S. 14. SAPMO DY 30 J IV 2/1/166, Protokoll 29. ZK-Plenum, Bl. $338 \mathrm{ff}$. Zu Oelßner auch Noziczka, Politik, S. 46, der allerdings einseitig zu Vieweg argumentiert und Oelßner davon positiv abgrenzt. Weitere Zusammenhänge bei Grieder, Sicht, $564 \mathrm{f}$., $573 \mathrm{f}$.

96 Diese Diskussion findet sich auch im PB-Protokoll vom 4. 12. 1956, das in ungewohnter Manier eine Entscheidung nicht einstimmig traf, sondern mit 6 zu 2 Stimmen gegen den Verkauf von leichten Traktoren an Einzelbauern votierte. Obwohl die Abstimmung nicht namentlich benannt war, kam eine Stimme sicher von Oelßner; vgl. Grieder, Sicht, S. 575 f. Die zweite könnte von Rau stammen. Seinen Vorschlag, den Räten der Bezirke und Kreise zu gestatten, in Ausnahmefällen leichte Traktoren an Einzelbauern zu verkaufen, akzeptierte das Politbüro als prüfenswert. SAPMO DY $30 \mathrm{~J}$ IV 2/2/518, Protokoll PB, 4. 12. 1956, Bl. $4 \mathrm{f}$. Der Kreis der Abstimmenden ist nicht klar festzulegen. Offenbar stimmte mindestens auch ein Kandidat des PB mit ab, denn es waren nur sieben Vollmitglieder an- 
Landwirtschaft in den größeren Kontext der Versorgungslage ein, welche die Irrealität des zweiten Fünfjahrplanes vor Augen führte. Als alarmierende Folge der wirtschaftlichen und politischen Krise wiesen Oelßner und Schirdewan auf die rapide ansteigenden Zahlen der Westabwanderung hin ${ }^{97}$. Auch ein Bericht der sowjetischen Botschaft an die KPdSU im Herbst 1956 empfahl eine Verlangsamung des Kollektivierungstempos; er brachte die Hoffnung zum Ausdruck, daß die „Ständigen Arbeitsgemeinschaften “ - für die in der DDR angeblich eine Initiative "von unten" vorhanden sei - die Voraussetzungen für eine künftige Bildung von LPG Typ I schaffen könnten. Aus sowjetischer Sicht hatte die SED ungerechtfertigter Weise die Bildung von LPG des Typs III forciert; auch habe die von der SED betriebene Überführung der LPG Typ I und II in solche des Typs III die Bauern völlig unvorbereitet getroffen ${ }^{98}$.

Die Probleme, die Vieweg und Langendorf behandelten, waren demnach in der Parteispitze und bei sowjetischen Stellen bekannt. Sie konnten durchaus hoffen, mit ihren Vorstellungen auf Interesse zu stoßen. Für den in allen Lebenslagen überaus ehrgeizigen Vieweg dürfte diese Aussicht auf Gehör eine nicht unwichtige Motivation bedeutet haben. Auch in der wissenschaftlichen Diskussion arbeitete das Duo nicht isoliert, sondern knüpfte an eine Debatte in der Agrarökonomik an. Asmus Petersen, ebenfalls Mitglied der Sektion Agrarökonomik der DAL, hatte das heikle Thema angeschnitten, inwiefern in westlichen Staaten ausgearbeitete betriebswirtschaftliche Methoden in sozialistische Landwirtschaftsbetriebe einfließen könnten ${ }^{99}$.

Das „Agrarprogramm“, für das Vieweg angeblich die praktische Seite beisteuerte und Langendorf die theoretische Fundierung übernahm, ging von der Überlegung aus, daß die Politik der Agrarumwälzung nicht gegen das Votum der Mehrheit der Bauern erfolgen könne, die die „seit 1952 durchgeführte Agrarpolitik als einen direkten bzw. indirekten Zwang zur Kollektivierung " sehe und daher den Aufbau des Sozialismus ablehne ${ }^{100}$. Um die Bauern jedoch von der Richtig-

wesend. Durchaus glaubhaft äußerte sich rückblickend Mückenberger vor der ZK-Abt. LW: „Nehmen wir den Genossen Oelßner an. Wie oft haben wir uns im Politbüro und im ZK mit ihm herumgestritten. Er sagte, man kann keine Genossenschaften bilden, wenn sie keine Perspektive haben, man kann keine Genossenschaften bestehen lassen, die den Wert von 7. - je AE [Arbeitseinheit] nicht erreicht haben." SAPMO DY 30 IV 2/7/75, Protokoll ZK-Abt. LW, 26. 1. 1959. Landwirtschaftliche Diskussionen mit „verschiedenen Meinungen“ im obersten SED-Führungszirkel erwähnt auch Schirdewan in seiner unveröffentlichten Stellungnahme vom 1. 1. 1958, explizit zu folgenden Themen: Abschaffung der Ablieferung, Traktoren für werktätige Einzelbauern, Fragen der Entwicklung der LPG, der MTS-Tarife und der Besteuerung der kleinen Warenproduktion. Bretschneider u.a., Karl Schirdewan, S. 502.

97 Siehe dazu Grieder, Sicht, S. 577. Zu Schirdewan: Skyba, Hoffnungsträger, S. 342-357, 367.

98 International Department, Rolle 109 (RGANI, 5-26-427, Bl. 178-187), Puschkin an ZK der KPdSU, Bericht über Entwicklung einfachster Formen der Kooperativen in der Landwirtschaft der DDR (erstellt von Kulikow), 10. 10.1956.

99 Scholz, Bauernopfer, S. 184 f.; Kluge, Affäre, S. 207 f.; vgl. auch Vieweg, Beitrag zur Ausarbeitung der Ökonomik der LPG, in: Einheit, September 1959.

100 Das folgende nach SAPMO DY 30 IV 2/4/396, Bl. 4-22, veröffentlicht von Scholz, Vie- 
keit des Weges zum Sozialismus zu überzeugen, müsse man ihnen eine Perspektive anbieten, die auf dem Prinzip beruhen sollte, daß in der DDR noch über einen langen Zeitraum beide Existenzformen, Privatbetrieb und Genossenschaft, nebeneinander bestehen bleiben. Das Konzept setzte auf wirtschaftlich tragfähige mittelbäuerliche Familienwirtschaften ohne Lohnarbeitskräfte. Diese Wirtschaften mit bis zu 40 ha LNF müßten daher voll mechanisiert werden. Da die Vorschläge keineswegs antisozialistisch motiviert waren, sollten explizit keine "großbäuerlichen" Betriebe entstehen, die nach Viewegs Sicht weniger durch ihre Hofgröße als durch die Beschäftigung von Lohnarbeitskräften definiert waren. Durch Maschinisierung und eine Übergangsregelung zu Anteilseignerschaften von Arbeitskräften sollte das kapitalistische Prinzip der Lohnausbeutung durchbrochen werden. Statt außerökonomischem Zwang, der „unzweckmäßig und hemmend“ gewirkt hätte, baute Vieweg auf wirtschaftliche Anreize und Hebel, um in kurzer Zeit einen hohen Leistungsstand der Agrarbetriebe zu erzielen.

Als Maßnahmen sah das Konzept erstens vor, das System der Pflichtablieferung nach einer Übergangsfrist abzuschaffen und zu einem einheitlichen Preissystem überzugehen; letzteres sollte Einflußfaktoren der Angebotsseite wie Witterung und Ernteergebnisse, aber auch gesellschaftliche Wünsche der Nachfrageseite flexibel berücksichtigen - eine Preisbildung auf dem freien Markt schied aus. Zweitens versprach es den Einzelwirtschaften eine unbeschränkte Versorgung mit Produktionsmitteln. Hierfür erwog es eine Wiederbelebung des gleichgeschalteten Genossenschaftswesens, z. B. in Form der Wiedereinrichtung von Maschinengenossenschaften, und ein Abrücken von bisher diskriminierend wirkenden Prinzipien, wie etwa eine an der Größe der landwirtschaftlichen Nutzfläche ausgerichtete Besteuerung. Drittens forderten Vieweg und Langendorf die Rückkehr zu einem pluralen Genossenschaftswesen, in dem die VdgB(BHG) eine echte Handelstätigkeit übernehmen sollte, die Bauern durch individuelle Gewinnbeteiligung für die landwirtschaftlichen Genossenschaften gewonnen werden und Spezialgenossenschaften wieder entstehen konnten. Viertens sollten die LPG unterstützt werden, damit sie schneller eine hohe Rentabilität erreichten. Die LPG dürften nicht mehr durch staatliche und Parteiorgane "bevormundet" werden. Neben den LPG sollten Kooperationsformen niedrigeren Vergesellschaftungsgrades eingerichtet werden, sogenannte „Bäuerliche Genossenschaftsbetriebe“; sie waren als Angebot für jene Bauern gedacht, für die ein LPG-Beitritt nicht in Frage kam. Fünftens empfahl das Konzept, die bisherige Form der MTS aufzulösen und sie in Maschinengenossenschaften umzuwandeln, bei denen die staatliche Beteiligung gleichwohl überwiegen würde, die als Lohnbetriebe aber ohne Subventionen auszukommen hatten. Während ein Teil der Maschinen in das Inventar der LPG einflieBen sollte, standen die Dienstleistungen der Maschinengenossenschaften sowohl den Produktionsgenossenschaften als auch den Einzelbauern zur Verfügung. Der letzte Punkt betraf die volkseigene Landwirtschaft und war am wenigsten aussagekräftig. Die Flächen der ÖLB sollten nicht mehr den LPG zugeschlagen werden und deren Rentabilität mindern, sondern waren ausschließlich dem volkseigenen

wegs alternative Agrarpolitik, S. 80-87; Scholz, Bauernopfer, S. 189 f.; eine kritische Würdigung bei Kluge, Affäre, S. 208-212. 
Sektor zuzuführen. Als Anreiz erwog das Papier, die dort beschäftigten Landarbeiter durch eine Gewinnbeteiligung zu motivieren.

Diese Vorstellungen wurden am 12. November 1956 in einer verkleinerten Parteigruppe an der DAL diskutiert, nachdem sich Vieweg offenbar durch die Vorgänge in Ungarn und Polen bestärkt fühlte. Er gab vor, an einer Konzeption für das Politbüro zu arbeiten, die noch streng geheim sei ${ }^{101}$. Im Unterschied zur Fassung des "Agrarprogrammes“ erklärte er in Gesprächen, daß ein Großteil der LPG vor der Auflösung stehe. Durch Informanten in der Parteigruppe erhielten das MfS und das ZK der SED umgehend Kenntnis über diese Gespräche in der DAL ${ }^{102}$.

Die Konzeption geriet durch die parallel verbreitete sogenannte „Plattform“ Wolfgang Harichs noch stärker in den Verdacht des "Revisionismus“103. Aus einem ganz anderen, nämlich auf Gesamtdeutschland bezogenen Blickwinkel heraus, forderte Harich den langfristigen Erhalt einer Mittelschicht in der DDR, wozu auch die Einzelbauern zählten, um auf dieser gesellschaftspolitischen Basis eine Einigung mit Westdeutschland erzielen zu können. Hinsichtlich der Agrarstruktur bedeutete dies, eine vollständige Sozialisierung zu unterlassen, diese nur so weit zu führen, daß sie keinerlei Ablehnung in der Bundesrepublik hervorrief 104 .

Im einzelnen forderte Harich die Aufhebung der Pflichtablieferung, statt dessen sollte ein nach Größenklassen differenziertes Steuersystem sozialen Ungleichheiten gerecht werden. Das implizierte den Fortbestand möglichst rentabler Einzelbauernwirtschaften. Harichs „Programm des besonderen deutschen Weges zum Sozialismus“ umfaßte die Forderung, daß die Bauernpartei „in Zukunft die unmittelbaren Interessen der Bauern gegenüber den Gesamtinteressen der Gesellschaft, die vom Staat wahrgenommen werden, energischer als bisher vertreten" müsse. Die Beschlüsse von Bauernkonferenzen seien von den Volksvertretungen aufzugreifen und "nach Möglichkeit" in Gesetzvorhaben einzubringen. Dies war bis dahin teilweise schon praktiziert worden, allerdings hatte die SED die eingebrachten Vorschläge im nachhinein stets als ihr eigenes geistiges Eigentum ausgegeben. Harich, der bekanntlich dem Kommunismus treu ergeben blieb, formulierte bezüglich des Blocksystems keine wesentliche Änderung. Obgleich die Blockparteien stärker als bisher eine Transmission der Wünsche der Bevölkerung von unten nach oben wahrnehmen sollten, ließ er an der Führungsrolle der SED keine Zweifel aufkommen. In der von ihm geforderten „Demokratisierung“ mußte die SED die Initiative ergreifen, daher sei den „Demokratisierungsbestrebungen, die von den Blockparteien ausgehen, durch eigene Maßnahmen de[r]

101 Scholz, Bauernopfer, S. 189.

102 SAPMO DY 30 IV 2/7/78, Bl. 386 f., Protokoll Sektorenleiterbesprechung, 4. 12. 1956, Informationen von Parske über die "Plattform Vieweg zu den Fragen der Agrarpolitik“.

103 Vgl. Scholz, Bauernopfer, S. 187. Ob ein Zusammenhang zwischen Vieweg und Harich besteht, ist ungeklärt.

104 Ebd., S. $186 f$. 
Wind aus den Segeln" zu nehmen ${ }^{105}$. Harichs Vorschlag bewegte sich völlig innerhalb des Herrschaftssystems.

Ohne fundamentale Herrschaftskritik zu üben, kamen auch im Ministerium und in der VdgB(BHG) Debatten in Gang. Die VdgB war gemäß einem Politbürobeschluß vom August 1956 dazu aufgefordert worden, Vorschläge einzureichen, welche Maschinen sie für geeignet hielt, um sie an Einzelbauern weiterzugeben ${ }^{106}$. Vor der Diskussion in der DAL über die Vieweg/Langendorfsche-Konzeption unterbreiteten die $\mathrm{VdgB}(\mathrm{BHG})$ und das Ministerium der neu konstituierten Agrarkommission des ZK der SED eine Reihe von Vorschlägen, die sehr ähnliche Elemente enthielten ${ }^{107}$. Wie bei allen eingebrachten Vorlagen blieb der besondere Beitrag der Zentralen Agrarpolitischen Kommission der DBD hier erneut ungenannt. Sie hatte sich im Oktober 1956 mit ihrem Vertreter im Zentralvorstand der VdgB, Herbert Eichhorn, darauf verständigt, gemeinsam Ideen auszuarbeiten, die dann in die VdgB- und Ministeriumspapiere einflossen, dort jedoch nicht mehr unter dem Siegel der DBD firmierten ${ }^{108}$.

$\mathrm{Zu}$ den Vorschlägen gehörte die Erweiterung der Kompetenzen der VdgB im Landhandel auf Kosten der nur unzulänglich arbeitenden Kreiskontore. Während sich Reichelt und Scholz sowie für die VdgB Biering und Broz für diese Änderungen aussprachen, hielten die strammen SED-Genossen Steffen, Grüneberg, Warnke, Quandt und Mellentin eine „Erweiterung der Handelsaufgaben der $\mathrm{VdgB} / \mathrm{BHG}$ nicht für richtig, da die VdgB/BHG dann immer mehr den Charakter einer politischen Massenorganisation verliert und von ihren politischen Hauptaufgaben abgedrängt wird" 109 . Auffälligerweise stimmte die Agrarkommission des ZK mehrheitlich dafür, von bestimmten Maschinen eine höhere Anzahl herzustellen und sie sowohl an die "Ständigen Arbeitsgemeinschaften“ als auch an Einzelbauern zu verkaufen. Die Genossen gestanden ein, daß die MTS über zu geringe Kapazitäten verfügten, um die Einzelbauern ausreichend zu bedienen. Außerdem hatte die vorangegangene Analyse ergeben, daß die DDR-Landwirtschaft hinter derjenigen der Bundesrepublik zurückblieb, wo auch die Industrie kontinuierlicher wachse. In Westdeutschland würde sich das Preisniveau - und damit, als entscheidendes Kriterium, der Wohlstand der bäuerlichen Produzenten - stärker zugunsten der Landwirtschaft verschieben, allerdings sei auch dort die Arbeitskräftesituation in der Landwirtschaft von Überalterung und der Abwanderung junger Menschen geprägt.

Der ZK-Abteilungsleiter Landwirtschaft Mellentin stellte Anfang Dezember auch im Ministerium eine Reihe von Ansichten fest, die seiner Meinung nach völlig verfehlt waren. So vertrete die Hauptabteilung LPG, geleitet vom stellvertretenden Minister, Genossen Neu, die Auffassung, die MTS in ihrer jetzigen Form

105 Harich, Schwierigkeiten, S. 139 ff., Zitate S. 146, 139.

106 SAPMO DY 30 J IV 2/2/496, Protokoll PB, 28. 8. 1956.

107 SAPMO DY 30 IV 2/7/26, Bl. $34 \mathrm{ff}$., Sitzungsprotokoll der LW-Kommission des ZK, 10. 11. 1956, hier Bl. 37-42. Zur Kommission wurden hinzugezogen die Regierungsvertreter Reichelt und Scholz sowie Mellentin, Streit und Broz.

108 ACDP VI-052-319, Protokoll Präsidium, 30. 10. 1956.

109 SAPMO DY 30 IV 2/7/26, Bl. 34 ff., Sitzungsprotokoll der LW-Kommission des ZK, 10. 11. 1956, Zitat Bl. 36. 
aufzulösen, ihre Maschinen den LPG zu übergeben und die MTS in Reparaturwerkstätten zu verwandeln - so hatte es auch das Vieweg-Papier vorgesehen. Die Hauptabteilung Pflanzliche Produktion befürwortete den Abzug der sowjetischen Berater im Ministerium, und die Hauptverwaltung VEG plädierte sogar für eine Abschaffung der Planung in der Land wirtschaft ${ }^{110}$.

Schon im März 1956 hatte sich Paul Scholz um Kontakt zu sowjetischen Stellen bemüht. Er versuchte, das Gewicht der DBD zu erhöhen, indem er dort unterstrich, wie notwendig eine bessere Einbindung von Funktionären außerhalb der SED sei ${ }^{111}$. Er kritisierte gegenüber dem Sekretär der sowjetischen Botschaft Kulikow, wie unzulänglich die SED die staatliche Leitung der Agrarwirtschaft organisiere. Sie treffe wichtige Entscheidungen zur Landwirtschaft völlig eigenmächtig ohne Hinzuziehung der zuständigen hochrangigen staatlichen Amtsinhaber oder Konsultierung anderer Parteien wie der DBD; sogar die SED-nahe Massenorganisation $\mathrm{VdgB}(\mathrm{BHG})$ bleibe ausgegrenzt. Er fühle sich als stellvertretender Vorsitzender des Ministerrates für Landwirtschaft zum „Hilfsfunktionär“ degradiert ${ }^{112}$.

Nonkonforme Ansichten zur Agrarpolitik wurden im Herbst 1956 auch in der DBD vertreten. Da deren Hauptverfechter Generalsekretär Rose war, ist davon auszugehen, daß diese Auffassungen im Sekretariat und Apparat des Parteivorstandes weitaus verbreiteter waren, als im einzelnen nachgewiesen werden kann $^{113}$. Hatte Goldenbaum nach seinem Auftritt auf dem IV. Parteitag 1955 noch herbe Kritik von Schnitzler und Rose im Sekretariat und aus der Arbeitsgruppe Materns einstecken müssen, so holte er nun zum Gegenschlag aus. Im Jahr 1958 streute Goldenbaum, dem Ulbrichts Aburteilung der innerparteilichen Opposition vor Augen stand, unter den Sekretären das - völlig übertriebene - Gerücht, "auch wir haben einen Schirdewan“. Goldenbaums Position in der Partei litt darunter, daß bis 1958 der Kontakt zum ZK allein auf Rose übergegangen war, der außerdem über gute Verbindungen zu Grotewohl und Mückenberger verfügte. Er begründete die schweren Anschuldigungen gegen Rose mit dem Vorwurf, dieser

110 SAPMO DY 30 IV 2/7/78, Bl.386f., Protokoll Sektorenleiterbesprechung, 4. 12. 1956.

111 International Department, Rolle 109 (RGANI, 5-26-427, Bl. 89-94), Gespräch Scholz mit Kulikow am 13. 3. 1956, 26. 3. 1956. Aus den Akten 425 und 426 (ebd., Rolle 109) ist ersichtlich, daß Botschaftsangehörige im Frühjahr 1956 systematisch Gespräche mit Vertretern von Massenorganisationen (Namokel, Schirdewan) führten, aber auch mit Burkhardt aus der Leitung der ZK-AG BO, ebd., Rolle 110 (RGANI, 5-28-429, Bl. 89-92, Gesprächsnotiz, 20. 3. 1956).

112 Als Beispiele führte er die vom MR beschlossene Neuregelung der Sozialversicherung an; weiter berichtete er über den desaströsen Zustand der LPG. Er beschwerte sich auch darüber, daß er bis heute nicht wisse, warum er als Minister von dem seiner Meinung nach minderqualifizierten Reichelt abgelöst worden sei. Mückenberger pflege keinerlei Kontakt zu ihm.

113 Als Indiz dafür spricht auch, daß in der fraglichen Zeit im Herbst 1956 keine aussagekräftigen Sekretariatsprotokolle vorliegen, bestenfalls sind einzelne Vorlagen und eine Tagesordnung überliefert; siehe ACDP VI-052-0533. Insofern die Belege für Roses Einstellung nur auf Aussagen Goldenbaums basieren, ist zu beachten, daß dieser 1957/58 in der Wahl seiner Mittel im Machtkampf mit Rose nicht zimperlich war. BStU MfS AP 661/92, Goldenbaum, Bl. 47-49, Treffbericht IM „Bernd“ (= Helmschrott), 5. 3. 1958. 
habe fraktionelle Sitzungen mit ausgesuchten Sekretären abgehalten - da dies häufiger vorkam, brachte er insofern ein schwaches Argument vor. Als „harte“ Belastungspunkte führte er an, Rose habe während des Aufstandes in Ungarn dafür plädiert, das Wort LPG aus dem Schulungsprogramm der DBD zu streichen Goldenbaum habe dies vereitelt -; vor allem aber habe Rose sämtliche unrentablen LPG auflösen wollen, wovon Rose die Hälfte aller LPG betroffen sah ${ }^{114}$. Außerdem habe der Widersacher das Bild der Sowjetunion und die Idealisierung der sowjetischen Bevölkerung kritisiert, wie sie in der DDR betrieben würde. Obwohl diese massiven Anschuldigungen aus der Feder jener IM stammten, die zu dieser Zeit eindeutig der Gruppe um Goldenbaum im Sekretariat zuzurechnen waren - auch der Führungsoffizier nahm dies zur Kenntnis -, ist ihnen durchaus ein Wahrheitsgehalt beizumessen ${ }^{115}$.

Rose erlaubte sich auch in den folgenden Jahren eine eigene Meinung zu den Anforderungen, die die Arbeitsgruppe BO an die DBD stellte. Im Herbst 1956 hatte die SED die Weisung ausgegeben, nun die „Ständigen Arbeitsgemeinschaften“, die gewünschten Vorstufen der LPG Typ I, besonders zu propagieren. Entgegen der Anweisung der Arbeitsgruppe BO lehnte es Rose ab, dieses Thema auf der Parteivorstandssitzung zum Hauptgegenstand seines Vortrags zu machen, und wiegelte die Genossen mit der Begründung ab, die DBD habe sich damit schon genug befaßt; außerdem sei diese Frage erst zur Frühjahrsbestellung wieder relevant ${ }^{116}$. Ende 1957 verweigerte Rose gegenüber der Arbeitsgruppe, sich auf einer Parteivorstandssitzung zusätzlich mit der Jugendweihe zu befassen; die DBD sei mit der Propagierung der LPG schon vollauf beschäftigt, so Rose ${ }^{117}$. Ihn trieb nicht prinzipielle Gegnerschaft zur Jugendweihe, vielmehr die zweckrationale Einsicht, die DBD in der ohnehin sehr schwierigen ideologischen Agitation auf dem Land nicht durch diese Überforderung lahmzulegen.

Sowohl die Eigenständigkeitsbemühungen für die DBD als auch die Absage, die DBD zum Werkzeug der rigideren antikirchlichen Politik Ulbrichts zu machen, ließen Rose in den Augen der SED jedoch verdächtig erscheinen und rückten ihn in die Nähe Schirdewans. Letzterer hatte sich bekanntlich dafür ausgesprochen, den Blockparteien eine größere Oppositionsrolle einzuräumen, und hatte auch einen weniger konfrontativen kirchenpolitischen Kurs vertreten, als ihn Ulbricht vorgab ${ }^{118}$. Es ist daher kein Zufall, daß Goldenbaum, der große Teile des Sekretariates und des Parteivorstandes hinter sich wußte, ab Sommer 1958 Roses Absetzung offensiv betrieb, zu einem Zeitpunkt, als die SED den Umgang

114 So IM „Bernd“, dessen Zuverlässigkeit als Quelle hoch ist. Ebd., Bl. 58, Bericht vom 19. 8. 1958.

115 Denn Materialien anderer Provenienzen, aus der DBD-Überlieferung und der AG BO, bestätigen diese Einschätzung. Ebd., Bl. 44 f., Bericht von IM „Bernd“ mit Bezug auf 1956, 3. 3. 1958. Ebd., Bl. 58, Bericht von IM „Bernd“, 19. 8. 1958. Alle Anschuldigungen, die IM „Bernd“ gegen Rose anführte, sah das MfS durch GI „Fuchs“ (= Böhm) bestätigt. Ebd., Bl. 62-72, Bericht GI „Fuchs“, 24. 11. 1958, B1. 68.

116 SAPMO DY 30 IV 2/15/2, Bericht AG BO an Ulbricht, 8. 8. 1957.

117 BStU MfS AP 661/92, Goldenbaum, Bl. 62-72, Bericht IM „Fuchs“, 24. 11. 1958, hier Bl. 68. Vgl. ACDP VI-052-0528, Protokoll Arbeitssekretariat, 1. 2. 1955.

118 Grieder, Sicht, S. 578 f., 592. 
mit solchen Kritikern öffentlich vorexerziert hatte. Bis Anfang 1958 waren die Auseinandersetzungen in der SED-Führungsspitze kaum an die Öffentlichkeit gelangt. Goldenbaum hatte 1956 seinerseits intern kritisiert, daß Ulbricht in der SED einen Personenkult betreibe. Inspiriert durch einen Chinaaufenthalt im Frühjahr 1956, interessierte sich Goldenbaum für Unterschiede zwischen dem chinesischen und sowjetischen Kommunismus, insbesondere in der Frage, wie man Kader zur „Bescheidenheit“ erziehe ${ }^{119}$.

Abgesehen von der Weigerung Roses, den Referatsvorgaben nachzukommen, erhielten das MfS und die Arbeitsgruppe BO offenbar erst 1958 umfassende Kenntnis vom Ausmaß seiner eigenständigen Überlegungen. Erst dann glückte es Goldenbaum, unter nachweislicher Mithilfe des MfS, Rose zunehmend ins Abseits zu drängen. Als Georg Böhm wegen seiner hartnäckig geleugneten NS-Vergangenheit ins Visier der SED geriet, hielt Goldenbaum ihm retrospektiv seine Mithilfe bei der Entmachtung Roses zugute ${ }^{120}$. Aus taktischen Gründen ließ die Arbeitsgruppe BO die Kontrahenten im Sekretariat agieren. Aus der Kombination von IM-Berichten und dem regulären Kontakt der Arbeitsgruppe mit dem Generalsekretär ergab sich ein doppelter Einblick in die DBD, ließ sich ihr Zustand besser einschätzen. In der Gegnerschaft der beiden Gruppierungen dürfte die Arbeitsgruppe BO sogar den Vorteil gesehen haben, das Engagement der Sekretariatsmitglieder für die Parteiarbeit zu erhöhen. Innere Machtkämpfe begünstigten im Endeffekt den Zugriff der SED, weil beide Gruppierungen versuchten, sich über die SED mehr innerparteilichen Einfluß zu verschaffen.

\section{Reaktionen: Zwischen offizieller Ablebnung und Interesse der Mitglieder}

Die Reaktion der SED auf den unangepaßten agrarpolitischen Reformdiskurs hatte verschiedene Facetten. Erstens wurden Vieweg und Langendorf einer immer härteren öffentlichen Kritik ausgesetzt: Vor allem auf Betreiben Mückenbergers, den Mellentin unterstützte, hatte das Politbüro am 15. Januar 1957 beschlossen, die Angelegenheit an die ZPKK zu übergeben. Viewegs angebliche „Revision des Agrarprogramms der Partei“" wurde auf dem 30. ZK-Plenum zusammen mit einer Reihe anderer „revisionistischer" Strömungen als „konterrevolutionär" verurteilt. Deshalb flohen die beiden am 26. März 1957 in die Bundesrepublik ${ }^{121}$. Nachdem es dem MfS mittelbar gelungen war, Vieweg am Zufluchtsort zu überzeugen, daß ihn ein faires Parteiverfahren erwarte - zu diesem Zweck hatte man seine langjährige Ehefrau zu ihm entsandt -, kehrten er und Langendorf im Herbst 1957 in die DDR zurück. Langen Verhören durch die Geheimpolizei folgte schließlich ein zwischen MfS und Justiz abgesprochenes Strafverfahren ${ }^{122}$.

119 BStU MfS AP 661/92, Goldenbaum, Bl. 44-46, Bericht IM „Bernd“, 3. 3. 1958. Zur China-Reise, ACDP VI-052-0316, Protokoll Präsidium, 10.1.1956.

120 BStU MfS AP 661/92, Goldenbaum, Bl. 140 f., betr.: Verhalten Ernst Goldenbaums gegenüber Georg Böhm, 22. 5. 1963.

121 Scholz, Bauernopfer, S. 194-197, 202-207.

122 Zur Verurteilung genauer Beckert, Instanz, S. 217 f.; Scholz, Bauernopfer, S. 212; Werkentin, Strafjustiz, S. 94; das Urteil wurde vom Landgericht Berlin am 27. 12. 1990 aufgehoben, dazu: Rehabilitierung von Prof. Kurt Vieweg und Marga Langendorf; Langendorf 
Zuvor hatte Ulbrichts Adlatus Hermann Matern auf einer Sitzung des Demokratischen Blocks im Februar 1958 Fred Oelßner wegen seiner „rückschrittlichen" Auffassung zu den LPG angegriffen ${ }^{123}$. Das Forum, vor dem er dies verkündete, mußte bei den Blockparteien jede Spekulation über Handlungsalternativen zum sozialistischen Kurs in der SED-Agrarpolitik ersticken. Die ZK-Abteilung Landwirtschaft hatte Anfang 1957 auch Minister Reichelt eingeschärft, daß die Auffassungen im Ministerium zur Frage der MTS eindeutig der offiziellen Linie der SED folgen müßten 124 . Die öffentliche Verurteilung Viewegs wegen seiner Opposition zu den MTS, deren Mängel auf der Hand lagen, verengten daher zugleich die künftigen Handlungsoptionen der SED.

Die SED bekräftigte ihren bisherigen, am Ziel des Sozialismus ausgerichteten agrarpolitischen Kurs. Während sie zwar bemüht war, Mißstände auszuräumen, ließ sie keine Zweifel darüber aufkommen, daß Veränderungen grundsätzlicher Art keine Option darstellten. Die Kenntnis über das ganze Ausmaß der Mißwirtschaft im Agrarwesen, zu der auch der unverdächtige SED-Apparat, wie die ZKAbteilung Landwirtschaft, die Agrarkommission oder die Staatliche Plankommission gelangt war, sowie der Verbreitungsgrad der Debatte bestärkten die SEDFührung darin, sich in manchen, politisch ungefährlichen Punkten flexibel zu zeigen. Daher machte sich das Regime das reformerische Potential dort, wo es ihr ökonomisch hilfreich erschien, um ihre Herrschaft abzufedern, durchaus zunutze ${ }^{125}$. Man nahm einige wirtschaftliche Modifizierungen vor. Beinahe schon beschlossene Benachteiligungen von Einzelbauern, wie sie etwa die Neuregelung der Sozialversicherung vorgesehen hatte, wurden vorerst zurückgestellt ${ }^{126}$.

Ungeachtet aller Eigenheiten, die Rose an den Tag gelegt hatte, konnte sich die $S E D$ voll auf die $D B D$ stützen. In öffentlichen Inszenierungen, etwa anläßlich der V. LPG-Konferenz im Frühjahr 1957, attackierten ausgesuchte Parteimitglieder, die etwa ihr Leid als vormalige Landarbeiter bei „Großbauern“ von ihrem glücklichen Dasein als Neubauern und LPG-Bauern abhoben, die Viewegsche Konzeption von 40 ha-Höfen in aller Schärfe ${ }^{127}$. Der V. Deutsche Bauerntag, der vor allem die Mitglieder der VdgB und damit große Teile der parteilosen Bauernschaft versammelte, sprach sich im März 1957 eindeutig für den sozialistischen Umbau der Landwirtschaft aus. Der Bauerntag beschloß sicherheitshalber eine Umbenennung der VdgB, die bisherige Namenskomponente der Bäuerlichen Handelsge-

wurde nach 6 1/4 Jahren freigelassen, Vieweg kurz danach. Der Weg in die Freiheit war für ihn mit einer Verpflichtung als IM verbunden. Der Wiedereinstieg in die akademische Gesellschaft fiel dem opportunistischen Lebenskünstler leicht. Dieser Charakterzug kommt in Scholz' Biographie zu kurz. Vgl. BStU MfS AIM 1200/77, I/1 Vieweg (= „Nordland“), Bl. 165, Bericht HA XVIII/6, 30. 12. 1964, über Gespräch mit Viewegs Frau.

123 Werkentin, Strafjustiz, S. 94.

124 Viewegs Berechnungen, wonach die jährlichen, nur durch die MTS verursachten Verluste bei 572 Mio. lägen, waren vom MLF bestätigt worden; SAPMO NY 4090/364, Bl. 335339, Mückenberger an Reichelt, 15. 1. 1957. Scholz, Bauernopfer, S. 198.

125 Vgl. SAPMO DY 30 J IV 2/2/518, Protokoll PB, 4. 12. 1956.

126 SAPMO DY 30 J IV 2/2/502, Protokoll PB, 9. 10. 1956, Bl. 4.

127 Bericht über V. LPG-Konferenz, „Bauern-Echo“, 3. 3. 1957. Auffällig ist die Zurückhaltung der DBD-Presse. Der Name Vieweg taucht im „Pflüger“ 1957 nicht auf. 
nossenschaften fiel künftig weg ${ }^{128}$. Diese Änderung dürfte ein Reflex auf Viewegs Vorschlag gewesen sein, gerade die Handelstätigkeit der $\operatorname{VdgB}(\mathrm{BHG})$ auszuweiten. Mit der VdgB hatte sich jene Gliederung öffentlich distanziert, an deren Spitze Vieweg einst als Generalsekretär gewirkt hatte, und in der die SED wegen der Mitgliedsstruktur wie auch aufgrund persönlicher Loyalitäten seine Parteigänger vermutete.

Die DBD bekam wieder einen vielfältigen Aufgabenkatalog zugewiesen. $\mathrm{Zu}-$ nächst sollte sie die Stimmung der bäuerlichen Bevölkerung ausloten und besänftigen. In der Hoffnung, zugleich politischen Mißmut aufzufangen, wurde ihr wie schon im Sommer 1953 gestattet, auf Kreisdelegiertentagungen im Frühjahr 1957 wirtschaftliche Wünsche ihrer Mitglieder zusammenzutragen. Parallel dazu sollte sie jedoch die politische Erziehung der Basis verstärken. Dazu wurden die Delegierungen von DBD-Funktionären auf SED-Parteischulen für die Ausbildung von Kadern für die Landwirtschaft ab Frühjahr 1957 sprunghaft erhöht ${ }^{129}$.

Zwar stufte die Arbeitsgruppe BO nur einen kleinen Teil der DBD-Mitglieder als "reaktionär" ein, aber immerhin die Hälfte als "schwankend“; knapp die Hälfte der Mitglieder sei der Partei und ihren Zielen treu ergeben ${ }^{130}$. Im Vergleich zu den anderen Parteien unterstrich die Arbeitsgruppe die hohe Quote von 60$70 \%$ Beteiligung an den Mitgliederversammlungen. Allerdings erreichte diese gerade im Herbst 1956 einen relativen Höchststand. Zweifellos ist dies auf das Interesse der Mitglieder an der damaligen agrarpolitischen Debatte, motiviert auch durch die Vorgänge in Polen und Ungarn, zurückzuführen, und nicht primär der Mobilisierungskraft der DBD zuzuschreiben.

Dieses im Vergleich zu den anderen Blockparteien positive Bild, das die Arbeitsgruppe von der DBD zeichnete, ist jedoch durch einen Blick in die Partei und an die Basis zu korrigieren. Zunächst verwundert es nicht, daß sich das Präsidium hinter Viewegs Verurteilung stellte ${ }^{131}$. Vieweg durfte in der Spitze der DBD kaum auf persönliche Sympathien hoffen, denn seine überhebliche Art, gepaart mit Ehrgeiz, der sich auf Kosten anderer zu profilieren suchte, hatte gerade die Bauernpartei während seiner Zeit als Generalsekretär der VdgB und ZK-Sekretär für Landwirtschaft unangenehm zu spüren bekommen. Unter den Mitarbeitern der DAL, die dazu beitrugen, aus dem „Agrarprogramm “ einen politischen „Fall“ $\mathrm{zu}$ konstruieren, ragen jedoch die SED-Genossen hervor. Die Mitglieder der DBD am Institut beteiligten sich anscheinend nicht an der Kampagne ${ }^{132}$.

Bei den einfachen Mitgliedern, die nichts von Viewegs Ränken gegen die DBD 1952 wußten, stießen seine Vorschläge verständlicherweise auf lebhaftes Interesse. Ohne die zeitliche und regionale Differenzierung der Stimmung und der bäuerlichen Forderungen hier im einzelnen anzuführen, bleiben im Ergebnis drei Komponenten festzuhalten, eine wirtschaftliche, eine politische und eine auf die Eigen-

128 Protokoll des V. Deutschen Bauerntages, S. 39; Graffunder, Dialektik, S. 117, 124.

12910 Personen wurden im April 1957 zur Schweriner SED-Schule geschickt. ACDP VI052-0320, Protokoll Präsidium, 24. 4. 1957.

130 SAPMO DY 30 IV 2/15/2, Bericht über Lage in der DBD, 12. 11. 1956.

131 ACDP VI-052-0320, Protokoll Präsidium, 12.2. 1957.

132 Zumindest konnten keine Hinweise in MfS- und SED-Überlieferung gefunden werden. 
ständigkeit der DBD bezogene. Im Umgang mit den Forderungen ist ersichtlich, daß diese zwar von den Kreisen an die Bezirke weitergegeben, schon in den Kreissekretariaten aber die unangemessen erscheinenden herausgefiltert wurden ${ }^{133}$. Eine Transmission der Interessen von unten nach oben blieb auf der ersten überlokalen Stufe stecken.

Sofern wirtschaftliche Forderungen nicht am Agrarkurs rüttelten, gab die DBD-Parteileitung solche an die SED weiter, insbesondere wenn damit organisatorische Verbesserungsvorschläge für den sozialistischen Teil der Landwirtschaft verknüpft waren. Entgegen der weitverbreiteten Kritik der DBD-Basis an den MTS, benutzte die Bauernpartei die Antragskommissionen der Bezirksparteitage dazu, eben jenes Kernstück der SED-Macht auf dem Lande, die MTS, zu stabilisieren: Während sie die Wünsche der Einzelbauern nach eigenen Kleinmaschinen unterschlug, drangen nur Bitten um eine bessere Maschinenausstattung der MTS zur mittelbaren Hilfe für die Privatbetriebe nach oben ${ }^{134}$. Gelegentlich nachzuweisende Forderungen nach Auflösung der LPG fanden kein Gehör ${ }^{135}$. Die DBD trug der SED nur noch zu, was ohne Furcht vor Sanktionen gesagt werden konnte. Längerfristig mußte diese affirmative Praxis einen enormen Problemstau verursachen.

Ein Hauptanliegen der bäuerlichen Bevölkerung betraf ein sozialpolitisches Thema, nämlich die Einbeziehung der Bäuerinnen ab dem 60. Lebensjahr in die Rentenversicherung sowie eine günstigere Beitragsstaffelung insgesamt ${ }^{136}$. Auf die besondere Mitgliederstruktur ist zurückzuführen, daß Neubauern eine Überprüfung des Einheitswertes und einen Schuldenerlaß für diejenigen Kredite, die nach 1950 gewährt worden waren, verlangten ${ }^{137}$. Nicht weitergeleitet wurden Vorschläge, die auf die Reduzierung von Ablieferungsverpflichtungen hinausliefen ${ }^{138}$. Auf wirtschaftlichem Gebiet fungierte die DBD also nur sehr bedingt als intermediäre Instanz.

Kein Medium war sie dagegen für die politischen Forderungen, die auf dem Land erhoben wurden. Die Bauernpartei, die Arbeitsgruppe BO und das MfS registrierten seit Mitte 1956 eine Fülle unangepaßter Verhaltensweisen, die sich mit den Ereignissen in Polen und Ungarn ausweiteten. Unter den Mitgliedern nähmen die "Schwankungen“ wegen der Vorgänge in den „Bruderländern“ zu. Man begegne dem sozialistischen Teil der Landwirtschaft mit wachsender Distanz und immer häufiger würden Begründungsmuster vorgebracht, die einen LPG-Eintritt hinauszögern sollten; die Ablieferung werde zurückgehalten und „negative Dis-

133 Zur selektiven Weiterleitung siehe Anträge an die Kreisdelegiertenkonferenzen 1957; ACDP VI-052-223/3.

134 ACDP VI-052-223/3, BV Cottbus, 1. 6. 1957, Vorlage Antragskommission an III. Bezirksparteitag.

135 BLHA Rep. 530, Nr. 2245, Bericht KL Nauen an BL der SED, 9. 7. 1956.

$136 \mathrm{Ebd}$.

137 Die Neubauernkredite waren 1950 als Wahlgeschenk erlassen worden. ACDP VI-052223/3, OG Zarchlin an KDK Lübz, 16. 2. 1957; ebd., Antrag KV Lübben an Bezirksparteitag, 14. 3. 1957.

138 Ebd., OG Forst, BV Cottbus, 1. 6. 1957, Vorlage der Antragskommission an III. Bezirksparteitag. 
kussionen“ würden in dem Verlangen nach "freier Wirtschaft“ gipfeln, wie die Arbeitsgruppe im Herbst 1956 meldete ${ }^{139}$. Die DBD-Mitglieder beanspruchten "freie Wahlen“ auch in der Variante, eine Abstimmung zwischen Vieweg und Mückenberger herbeizuführen.

Wie 1953 war die Stimmung aufgewühlt und zeugte von der Politisierung der ländlichen Bevölkerung. Frauen im mecklenburgischen Parchim drohten Funktionären, sie sollten ihre politischen Aktivitäten einstellen, sonst könne es sein, daß sie einst wie in Ungarn am Baum hängen würden. Während die einen hinter vorgehaltener Hand verbreiteten, „der Spitzbart müsse weg“, würden andere jedes Gespräch mit Funktionären prinzipiell verweigern. Eine Reihe von ansonsten als kompetent geltenden DBD-Funktionären legte ihre Ämter kommentarlos nieder. „Großbäuerliche“ Kreise wetterten, man solle die „Russen raushauen“. „Umsiedlerbauern" aus Schlesien hofften erneut, bald wieder in die Heimat zurückkehren zu können. Für andere Flüchtlinge und Vertriebene war das westdeutsche Lastenausgleichsgesetz ein Vorbild, das sie in die DDR übertragen sehen wollten ${ }^{140}$. Diejenigen Funktionäre der DBD, die sich als Protagonisten der Kollektivierung lokal profiliert hatten, standen unter Beschuß. So mancher Aktivist wurde nachts durch Pochen an die Haustüre aus dem Bett geschreckt und aufgefordert herauszukommen, das konterrevolutionäre Komitee aus Ungarn sei da, um ihn zu erschießen ${ }^{141}$.

Wie drei Jahre später während der Vollkollektivierung beharrten Kritiker darauf, wenn schon verstaatlicht würde, dann müsse dies auf klar ersichtlicher gesetzlicher Grundlage geschehen, „damit der Staat für alles allein verantwortlich gemacht werden kann"142. Damit stellte man die vorgebliche „Freiwilligkeit" bloß und charakterisierte die Kollektivierung als staatlich geplanten und administrativ durchgesetzten Akt. Ein zuverlässigeres Stimmungsbild als die Kreisdelegiertenanträge vermitteln Informationsberichte der DBD aus dem Jahr 1957. Vor allem in Regionen mit einem hohen Anteil an Einzelbauern, wie im sächsischen Dippoldiswalde, klammerten sich die DBD-Mitglieder an die Positionen von Vieweg, für den sie "durchs Feuer gehen" wollten, oder traten wie im Kreis Gadebusch für eine öffentliche Abstimmung über ihn ein. Anläßlich der bevorstehenden Volkskammerwahlen erhoben sie grundsätzliche Kritik am politischen System, an den Einheitslistenwahlen und am Fehlen einer parlamentarischen Opposition ${ }^{143}$. Im Kreis Meißen knüpften die Bauern ihren Urnengang an Bedingungen: „Ohne eine Sollabsetzung gehen wir nicht zur Wahl.“ Auch in den Kreisen Köthen, Rosslau und Wittenberg werde ,in ähnlicher Form die Wahlbeteiligung von der Erfüllung bestimmter Forderungen, wie z.B. Straßen- und Wohnungsbau, Schulneubau, Düngerzuteilung usw. abhängig gemacht“. Teile der ländlichen Gesellschaft gingen insofern einen Kompromiß mit der SED-Herrschaft ein, als sie gegen die Gewährung von Vergünstigungen die an sich verwor-

139 SAPMO DY 30 IV 2/15/2, Bericht über Lage in der DBD, 12. 11. 1956.

140 ACDP VI-052-010/2, Informationsbericht, 13. 12. 1956; vgl. Schwartz, Lastenausgleich.

141 Diese und andere Beispiele in ACDP VI-052-010/2, Informationsbericht, 20. 11. 1956.

142 SAPMO DY 30 IV 2/15/2, Bericht über Lage in der DBD, 12. 11. 1956.

143 ACDP VI-052-010/3, Abt. Org., 27.4. und 11. 6. 1957, Stimmungsberichte 7 und 8. 
fene Wahlpraxis nach der Einheitsliste beachten wollten. Nach der ersten Kollektivierungswelle war die ländliche Gesellschaft gespalten. Auf bestimmte Teile der DBD-Basis war als Stoßtruppe des Sozialismus auch 1957 Verlaß. So beobachtete man unter DBD-Mitgliedern eine Bewegung zu freiwilligen Leistungsverpflichtungen, „die aber in den Dörfern unter den übrigen Bauern noch keinen breiten Raum" einnehme ${ }^{144}$.

Als Ergebnis der Diskussion von 1956 drängten die Parteimitglieder aller Ebenen, ganz besonders jene, die den sozialistischen Kurs mittrugen, vehement darauf, den Eigenanteil der politischen Tätigkeit der DBD klarer als bisher bekanntzugeben. Die Parteivorstandssitzung im Dezember 1956 notierte neuerliche „Schwankungen“ in der Frage des Bündnisses mit der SED. Im „Bauern-Echo“ müsse stehen - so die Kollegen im Kreis Pößneck, aber auch in anderen Bezirken: „auf Vorschlag der Demokratischen Bauernpartei hat das Zentralkomitee der SED beschlossen“145. „Sowohl im Rechenschaftsbericht als auch in einzelnen Diskussionen zeigen sich solche Tendenzen, die auf Grund des 28. und 29. Plenums durchgeführten Maßnahmen zur Förderung der Landwirtschaft als Ergebnisse der Politik der DBD darzustellen."146 Dieses Beispiel aus Lübz illustriert die besondere Lage der DBD und die davon herrührenden Befürchtungen der SED um ihre "führende Rolle“ auf dem Land. „Schwankungen“ in der Bündnisfrage meinten nicht ausschließlich eine Abkehr von der SED-Politik, sondern man kanzelte mit diesem Wort das Selbstbewußtsein der DBD-Anhänger ab, die ihre Partei für eine der SED zumindest ebenbürtige sozialistische Kraft auf dem Lande hielten. Dies sollte sich, so forderte die Basis weiter, in einer höheren Anzahl von politischen Mandaten bemerkbar machen und in der Wiederzulassung von Hochschulgruppen der DBD ${ }^{147}$.

Auch in der SED-Berichterstattung an die sowjetischen Machthaber in Berlin 1956 wurde der Zustand der Blockpolitik und die Eigenständigkeitsbestrebungen der DBD thematisiert. Die sowjetischen Mitarbeiter rieten Ende 1956, die von der SED völlig mißachtete Blockpolitik wieder als Mittel der Politik zu begreifen ${ }^{148}$.

144 Ebd., Abt. Org., 27. 4. 1957, Stimmungsbericht 7.

145 ACDP VI-052-0160, Protokoll PV-Sitzung, 17./18. 12. 1956, aus Diskussionsprotokoll, S. 29 (Zitat); ACDP VI-052-0319, Protokoll Präsidium, 30. 10. 1956. Schon 1955 hatte eine Präsidiumssitzung festgestellt, daß die Mitglieder mehr Beachtung ihres politischen Beitrages erwarteten; ACDP VI-052-0315, Protokoll Präsidium, 13. 12. 1955. Das Sekretariat notierte mit Befremden, daß die Worte Mückenbergers auf der 23. ZK-Tagung zur DBD im ND nicht veröffentlicht worden waren; ACDP VI-052-0529, Protokoll Sekretariat, 21. 4. 1955. Zu 1957 siehe auch: SStAD BPA SED BL Dresden IV/2.15.005, Bericht SED-KL Bautzen, 14. 3. 1957, über KDK der DBD am 22. 2. 1957.

146 MLHA BPA Schwerin IV/2/15/1687, Bericht der SED KL-Lübz über die KDK der DBD, 23. 3. 1957.

147 ACDP VI-052-010/3, Abt. Org., 27. 4. 1957, Stimmungsbericht 7. ACDP VI-052-0160, Protokoll PV-Sitzung, 17./18. 12. 1956, Diskussionsprotokoll, S. 29.

148 International Department, Rolle 110 (RGANI, 5-28-429, Bl. 162-191), Über Lage in den demokratischen Parteien der DDR und über die Zusammenarbeit der SED mit diesen, von Gesandtschaftsrat S. Astawin, 11. 12. 1956, von Puschkin an ZK der KPdSU gesandt, bes. Bl. $190 \mathrm{f}$. Als symptomatisch für den Umgang mit den anderen Blockparteien wurde angeführt, daß Matern eine für Anfang November 1956 wegen der Ereignisse in Polen 
Die SED müsse die Zusammenarbeit verbessern, um die Basis der Blockparteien, die die SED nicht erreichen könne, für die gesamtdeutsche Arbeit zu gewinnen. Da die Existenz der Parteien dem Volk und dem Westen den demokratischen Charakter der DDR vorführen solle, würde eine Vernachlässigung des Blockes und der Nationalen Front die weitere „Demokratisierung“ der DDR erschweren, so die Analyse der sowjetischen Botschaft in Berlin.

Doch es sollte 1957 anders kommen. Ulbricht, die Arbeitsgruppe BO und das MfS zielten auf eine Beibehaltung des bisherigen Stils der SED-Herrschaft. Die Kontrolle gegenüber der DBD, die sich etwa in einer rigoroseren Kaderpolitik niederschlug, wurde intensiviert ${ }^{149}$. Die DBD gab auf dem V. Parteitag im September 1957 ihren bis dato zumindest propagierten, wenngleich nicht verfolgten, eigenständigen programmatischen Anspruch auf ${ }^{150}$. Anstelle der bislang geltenden Formulierung im Parteiprogramm, die die DBD als „selbständige politische Partei der werktätigen bäuerlichen Bevölkerung " charakterisierte, definierte eine Grundsatzerklärung die DBD nur noch banal als „erste demokratische Bauernpartei in der Geschichte Deutschlands “151. Die Parteitagsregie schloß mögliche Diskussionen darüber aus. Während die Arbeitsgruppe BO die DBD noch 1955 als „kleinbürgerliche“ Partei abgetan hatte, und die neue Charakterisierung als „demokratische Partei“ sie insofern im Rahmen der bündnisideologischen Doktrin aufwertete, war dies freilich nur ein vordergründiges Zugeständnis. Tatsächlich engte die Neuformulierung das politische Gewicht der Bauernpartei ein, so wie es die SED, voran Ulbricht, wünschte. Einen weiteren Anlaß für diesen nach außen proklamierten Verzicht auf ein eigenes Profil bildeten die Vorgänge im polnischen Parteienwesen 1956. Sogar die zaghaften und überaus verhalten vorgebrachten Versuche der DBD, die Wünsche ihrer Mitglieder in den politischen Prozeß einzubringen, wurden von der Arbeitsgruppe $\mathrm{BO}$ im nachhinein negativ ausgelegt. Diese warf der DBD in einem Bericht an Ulbricht 1958 vor, sie habe ihre Mitglieder erst spät für die LPG-Bildung gewonnen und lange auf eine „ökonomische Interessenvertretung der werktätigen Einzelbauern" gesetzt ${ }^{152}$.

und Ungarn angesetzte Zusammenkunft schlichtweg vergessen hatte - ohnehin würden nur sporadisch Treffen der Parteiführer stattfinden. Siehe auch ebd., Bl. 89-92, Gesprächsnotiz M.I. Boronin, 2. Sekretär der Botschaft, über Treffen mit Burghard [= Burkhardt], ZK AG BO, zur Arbeit mit anderen Parteien am 2. 3. 1956, 20. 3. 1956.

149 Beispiele für DBD-Staatsfunktionäre, die wegen mangelnder Unterstützung des sozialistischen Kurses auf Ablehnung der SED stießen, in SAPMO DY 30/vorl. SED 4071, $4072,4073,4074,5127,5128,5130$.

150 Reichelt, Blockflöte, S. 157 f., Graffunder, Dialektik, S. $138 \mathrm{f}$.

151 So festgelegt auf dem V. PT in Leipzig, 2.-4.7.1957, im Dokument „Charakter der DBD und Grundsätze ihrer politischen Tätigkeit“".

152 SAPMO DY 30 IV 2/15/2, Bericht der AG BO, Entwicklung der kleinbürgerlich-demokratischen Parteien in der Zeit zwischen dem IV. und V. Parteitag unserer Partei, mit Anschreiben an Ulbricht vom 31. 5. 1958. 


\section{Exkurs: Der Blick nach Polen 1956}

Ulbricht hatte 1956 unterstrichen, daß die Politik des „Tauwetters“ in Polen kein Vorbild für die Landwirtschaft der DDR abgeben könne. Er bezog sich sowohl auf die LPG-Auflösungen als auch auf die gleichzeitig erfolgte Aufwertung der polnischen Bauernpartei neben der Arbeiterpartei. Die polnische Vereinigte Bauernpartei (ZSL) spielte trotz ihrer Gleichschaltung 1950 weiterhin eine Rolle, da sie stärker in der Bevölkerung verankert war als die Arbeiterpartei. Die Gleichschaltung hatte die Partei in eine tiefe Krise gestürzt. Viele Mitglieder vermißten daraufhin eine programmatische Eigenständigkeit und erwarteten die Auflösung der Partei, wie das Politbüro der Polnischen Vereinigten Arbeiterpartei (PZPR) feststellte ${ }^{153}$. Der Vorsitzende der ZSL, Kowalski, der, obgleich er insgeheim der Arbeiterpartei angehörte, offenbar dennoch um eine gewisse Distanz zur PZPR bemüht war, konnte die ZSL in den fünfziger Jahren kaum stabilisieren ${ }^{154}$. Der Parteiaufwertung 1956 folgten bis 1959 Jahre begrenzter Eigenständigkeit ${ }^{155}$.

Ulbricht wies in einer internen Rede Ende 1956 die Beschlüsse des bahnbrechenden 8. Plenums der PZPR weit zurück, die SED werde keinesfalls diese „Fehler" begehen. Besagtes Plenum habe der ZSL die gleichen Rechte eingeräumt wie sie der PZPR zustünden, weshalb deren "führende Rolle“ verlorenzugehen drohe ${ }^{156}$. Eine ähnliche Würdigung der Bauernpartei oder anderer Blockparteien in der DDR schloß Ulbricht kategorisch aus ${ }^{157}$. Während Ulbrichts Ansinnen auf den weiteren Ausbau der SED-Vormacht hinauslief, vertrat sein Gegenspieler Schirdewan 1956 bekanntlich Konzeptionen, die eine Vitalisierung der Blockpolitik und damit der anderen Blockparteien implizierten, allerdings ohne damit die Prinzipien des politischen Systems grundlegend verändern zu wollen ${ }^{158}$.

Die DBD hatte lebhaftes Interesse an Kontakten zu ihrer polnischen Schwesterpartei159. 1953 war eine polnische Delegation als Gast auf dem III. Parteitag der DBD anwesend. Davor hatte es offensichtlich von seiten der SKK Bedenken gegen offizielle Kontakte zu den Bauernparteien in den benachbarten volksdemokratischen Ländern gegeben. Tschuikow bevorzugte bis 1953 eine gesamtdeutsche Ausrichtung der DBD, die nur Verbindungen mit westdeutschen Bauern opportun erscheinen ließ. Erst seit der „Zwei-Staaten-Doktrin“ 1955 durfte die DBD

153 Centrum władzy, S. 49f., Sitzung des Sekretariats des PB der PZPR, 30. 6. 1950; ebd., S. $62 \mathrm{f}$., Sitzung des Sekretariats des PB der PZPR, 9. 10. 1950.

154 Ebd., S. 168-170, Sitzung des PB der PZPR, 14. 6. 1956; Gespräch zwischen dem Sekretär des Exekutivkomitees der ZSL, A. Juszkiewicz, mit dem 2. Sekretär der sowjetischen Botschaft, A. P. Jermolow, am 26. 3. und 10. 4. 1952, in: Wostotschnaja Ewropa, Bd. 2, S. 703-710.

155 Paruch spricht von einer Phase die sich auf eine „Penetrierung“ beschränkte, ders., Politycy Zjednoczonego Stronnictwa Ludowego.

156 SAPMO DY 30 IV 2/13/197, Über die politische Lage in der Volksrepublik Polen (1956).

157 Ulbricht kritisierte die Entwicklung in Polen anläßlich einer erweiterten Sitzung des Präsidiums des ZK der KPdSU am 24. 10. 1956, zu der Vertreter der SED, KPTsch und aus Bulgarien anwesend waren. SSSR i Polscha, S. 187.

158 Als Leiter der Abt. LOPM hatte sich Schirdewan schon 1955 dagegen ausgesprochen, die DBD durch systematische Mitgliederabwerbungen zugunsten der SED zu schwächen; SAPMO DY 30 IV 2/5/192, Bl. 56, Schirdewan an Fritz Kleinert, 28. 11. 1955.

159 Reichelt, Blockflöte, S. 148-152. 
offizielle Kontakte nach Polen pflegen. Am Schweriner Bezirksparteitag 1955 nahm eine Stettiner Delegation der ZSL teil, und auch der IV. Parteitag der DBD 1955 begrüßte polnische Gäste ${ }^{160}$. Im März 1956 reiste eine Delegation der DBD unter Goldenbaums Leitung zum Parteitag der ZSL. Reichelt zufolge führte die DBD seit März 1954 interne Verhandlungen mit der ZSL, die im September 1956 in ein gemeinsames Kommuniqué mündeten ${ }^{161}$.

Das Verhältnis zur ZSL verbessere sich laufend, konstatierte das Protokoll der Präsidiumssitzung just im Monat der politischen Wende in Polen, im Oktober 1956; das Zusammentreffen 1955 war noch unterkühlt, "sachlich-diplomatisch“ verlaufen ${ }^{162}$. Auch Kreisverbände bemühten sich seit Ende 1956 um einen Austausch mit ihrer polnischen Schwesterpartei, was der Parteiapparat der SED mit Befremden registrierte ${ }^{163}$. Angesichts des Bedeutungsgewinnes der ZSL im polnischen Parteiengefüge und mit dem Abrücken von einem strikten Sozialisierungskurs in der Landwirtschaft mußten diese DBD-Initiativen die SED hellhörig machen. Die Versuche der DBD, durch auswärtige Kontakte an Profil als Partei zu gewinnen, riefen bei der SED daher heftige Ablehnung hervor. Die Instrukteure der Bezirksleitungen wurden anläßlich einer Beratung im ZK eigens angewiesen zu überprüfen, ob es in den Bezirken „Beziehungen der anderen Parteien zu kleinbürgerlichen Parteien der Volksdemokratien gibt", und dies an das ZK weiterzumelden ${ }^{164}$.

Die DBD gab sich in diesem Zusammenhang sichtlich Mühe, den Einblick der SED in Meinungsbildungsprozesse und Vorgänge zu begrenzen ${ }^{165}$. Anläßlich des V. Parteitages 1957 wurde eine gemeinsame Erklärung der Parteivorstände der deutschen und polnischen Bauernpartei erarbeitet. Wo die Probleme dieser Verlautbarung lagen, deutet Reichelt sybillinisch an: „Die Verhandlungen hierzu hatten sich als sehr kompliziert erwiesen, insbesondere die Stellung und Beziehungen zu den Arbeiterparteien, der Sowjetunion und zur Umgestaltung der Landwirtschaft. " ${ }^{166}$ Bis dahin war es der SED gelungen, das ursprüngliche Interesse der DBD am polnischen agrarpolitischen Weg als inakzeptabel zu brandmarken oder zumindest zu unterdrücken. Die Arbeitsgruppe BO schraubte 1957 das ideologische Anforderungsprofil für die DBD nach oben. Aufgrund des eingespielten Verhältnisses zwischen der Arbeitsgruppe und dem Sekretariat der DBD erscheint es plausibel, daß die Bauernpartei in diesem heiklen Punkt ähnlich wie

160 ACDP VI, unverz. Best. BV Schwerin, Protokoll Bezirksparteitag Schwerin, 5./6. 7. 1955; ACDP VI-052-0530, Protokoll Präsidium, 14. 9. 1955, Einschätzung des IV. PT.

161 Reichelt, Blockflöte, S. $149 \mathrm{f}$.

162 ACDP VI-052-0319, Protokoll Präsidium, 16. 10. 1956. Zitat Goldenbaum, ACDP VI052-0168, Protokoll PV, 20. 12. 1958.

163 Die Bitte von Karl Schoepke um Genehmigung für eine Polenreise im Februar 1957 wurde in Materns Apparat mit einem großen roten Fragezeichen quittiert. SAPMO DY 30/vorl. SED 4073, Schriftwechsel MdJ an ZK, 13. 3. 1957; Schoepke an MdJ, 13. 2. 1957.

164 BLHA Rep. 530, Nr. 2246, BL Potsdam über Beratung mit Instrukteuren der BL im ZK, AG BO, 15. 10. 1956.

165 Entsprechend schmal ist die DBD-Überlieferung hierzu, und entsprechend vage sind die Formulierungen dazu in den DBD-Protokollen gehalten, die in der Regel in SED-Hände gelangten.

166 Reichelt, Blockflöte, S. 150. 
bei den Diskussionen um das Parteiprogramm 1955 den Kontakt zu SED-Stellen suchte und zur Zufriedenheit der SED vorauseilenden Gehorsam an den Tag legte.

Eine Reihe von Indizien legt den Schluß nahe, daß die SED die DBD außenpolitisch dafür einspannen wollte, umgedreht die ausländischen Bauernparteien auf den sozialistischen Kurs in der Landwirtschaft einzuschwören. Hans Rietz berichtete dem MfS Ende Oktober 1957 darüber, daß in der Führung der ZSL „eine Säuberung durchgeführt wurde, die zugunsten des Fortschritts verlief"167. Obgleich gut informiert, konnte oder wollte er jedoch keine Auskunft darüber geben, in welcher Form eine Zusammenkunft mit der ZSL geplant sei. 1959 besuchte erneut eine Delegation der ZSL die deutsche Bauernpartei. Das Präsidium verbot der hauseigenen Presse, darüber zu berichten ${ }^{168}$. Die Arbeitsgruppe BO, die im Vorfeld offenbar über diesen Besuch nicht informiert worden war, kanzelte die Führungsspitze der DBD ab, Kontakte zur ZSL seien aus Sicht der SED unerwünscht. Sie stützte sich auf Informationen des MfS, das seit Juni 1959 versuchte, seine Informanten über die Kontakte des Parteivorstandes zur ZSL zu befragen ${ }^{169}$. So war dem MfS bekannt geworden, daß am 18. und 19. März 1959 zwei Mitarbeiter der polnischen Bauernpartei den Parteivorstand der DBD aufgesucht hatten und man einen Delegationsaustausch vorbereitete. Außerdem berichtete der Informant davon, daß in der ZSL insgesamt wie auch in der Besuchsdelegation zwei verschiedene „agrarpolitische Linien“ vertreten worden seien. Insbesondere die Zweifel am agrarsozialistischen Kurs riefen den heftigsten Widerspruch der Arbeitsgruppe hervor. Sie verbot der DBD-Spitze Kontakte zur ZSL zum damaligen Zeitpunkt ${ }^{170}$. In den sechziger Jahren benutzte die SED die Blockpartei DBD dazu, unter den Ostblockstaaten ihre Selbstdarstellung als führende Arbeiterpartei zu schärfen. Initiativen von Blockparteien aus dem benachbarten Ausland ließ sie durch deren Schwesterpartei in der DDR ausbremsen ${ }^{171}$.

167 Außerdem sei „die Spannung zwischen der bulgarischen und der polnischen Bauernpartei [...] beseitigt worden. " BStU MfS AP 14985/92, Rietz, Bericht der HA V/3 über Aussprache am 29. 10. 1957, Zitat Bl. 32.

168 ACDP VI-052-0539, Protokoll Präsidium, 1. 6. 1959.

169 BStU MfS AIM 392/83, I/1 Mundt, Bl. 27-29, Treffbericht mit Quelle GI „Irmgard“, Mitarbeiterin des PV, 4. 6. 1959. BStU MfS AIM 927/60, A-Akte Böhm, Bl. 93-95, 4. 7. 1959.

170 Ebd., Bl. 77, Bericht vom 23. 3. 1959.

1711967 startete die bulgarische Bauernpartei eine Initiative mit dem Ziel, eine internationale Vereinigung aller Bauernparteien ins Leben zu rufen. Den Vorsitz strebte der Vorsitzende der Bulgaren, Traikoff, an, zu dem Goldenbaum kein gutes Verhältnis hatte; so Reichelt, Blockflöte, S. 150f. Goldenbaum lehnte Traikoffs Vorhaben ab. Die Gründe dürften außerdem vor allem durch die Richtlinienkompetenz der SED bestimmt worden sein. Letzteres vermutete zumindest Traikoff nach SED-Berichten als eigentlichen Grund für Goldenbaums Ablehnung. Lt. Traikoff hinke die DDR damit jedoch der Zeit hinterher, denn er gab vor, sein Plan sei mit der KPdSU abgestimmt; SAPMO DY 30 IV A 2/2.023/185, Bericht Köhlers für Grüneberg, 5. 5. 1967. 


\section{Die Fortsetzung des sozialistischen Kurses Mitte 1957}

Bis zum Sommer 1957 hatte die SED den Einzelbauern aufgrund politischer Überlegungen während der Agrardebatte und angesichts knapper Ressourcen eine Verschnaufpause gegönnt, indem sie die Devise ausgegeben hatte, vorrangig die Bildung von "Ständigen Arbeitsgemeinschaften" zu betreiben. Diese Zwischenphase stabilisierte den überforderten genossenschaftlichen Teil der Landwirtschaft, da die MTS außerstande waren, die benötigte Maschinenleistung für eine ordnungsgemäße Bewirtschaftung zu erbringen. Sie entschärfte dadurch die finanzielle Belastung, die die Stützungszahlungen für LPG und MTS dem Fiskus aufbürdeten. Die ideologische Linie zur Neugründung von LPG Typ I wurde schon im Juli/August 1957 von der ZK-Abteilung Landwirtschaft wieder festgeklopft. Die Abteilung nahm damit die Ausrichtung vorweg, die die 33. ZK-Tagung im Herbst 1957 verkündete ${ }^{172}$.

Die "Ständigen Arbeitsgemeinschaften“, die dem Viewegschen Typus des „Bäuerlichen Genossenschaftsbetriebes“ zu nahe gekommen sein dürften, wurden nun als „falsche Philosophie" der VdgB verworfen, die diese noch im Frühjahr auf dem V. Deutschen Bauerntag unterstützt hatte ${ }^{173}$. Nun wurde die Bildung solcher Arbeitsgemeinschaften als bloßer Vorwand ausgelegt, nicht in die LPG eintreten zu wollen. Die rund 9000 Arbeitsgemeinschaften waren jedenfalls eine verlockende Ausgangsbasis für die Gründung von LPG.

Unterdessen kämpfte die Bauernpartei seit dem V. Parteitag (2. bis 4. Juli 1957) in Leipzig mit rückläufigen Mitgliederzahlen. Kaderleiter Rietz führte dies gegenüber dem MfS auf das Bekenntnis des Parteitages zum sozialistischen Weg in der Landwirtschaft und auf die Ausdehnung der LPG-Neugründungen zurück. In der Tat hatte die DBD in der ersten Jahreshälfte noch einen sehr hohen Mitgliederzuwachs verbucht; er ist als Indiz dafür zu werten ist, daß sich die einzelbäuerliche Bevölkerung, auf deren Werbung sich die Partei konzentrieren mußte, von der DBD eine Interessenvertretung erhoffte ${ }^{174}$. Von 7122 Neuaufnahmen 1957 fielen nur 2233 in die zweite Jahreshälfte, also nach der Bekräftigung des Sozialismuskurses auf dem V. Parteitag175.

Doch die ZK-Abteilung Landwirtschaft gab die Losung aus, den Sozialismus zu forcieren. Mückenberger warf der DBD im August 1957 vor, sie verwechsle das Prinzip der Freiwilligkeit bei der Bildung von LPG mit der „Selbstlauftheorie“ und drängte die Arbeitsgruppe $\mathrm{BO}$ zu einer schärferen Instruierung. Letztere

172 Schulz, „Kapitalistische Länder überflügeln“, S. 18 f.; erst in den Herbst 1957 datieren diesen Umschwung Nehrig/Noziczka, Weiterentwicklung, S. 1095, und Noziczka, Politik, S. 48-50.

173 Die unterschiedliche politische Einordnung der Arbeitsgemeinschaften innerhalb eines Jahres spiegelt sich auch in der agrarhistorischen Forschung der DDR wider: Graffunder, Dialektik, S. 117; Sommer, Entwicklung der landwirtschaftlichen Produktionsgenossenschaften, S. 242; Klemm u.a., Agrargeschichte, S. 196.

$174 \mathrm{Vgl}$. SAPMO DY 30 IV 2/15/2, AG BO an Ulbricht, 8. 8. 1957.

175 ACDP VI-052-160/7, Übersicht: Organisatorische Entwicklung 1957. BStU MfS AP 14985/92, Rietz, Bl. 31 f., Bericht der HA V/3 über Aussprache am 29. 10. 1957. SAPMO DY 30 IV 2/15/4, Protokoll Arbeitstagung Bezirk Neubrandenburg, 12. 12. 1957. 
holte sogar bei Ulbricht die Rückversicherung dazu ein ${ }^{176}$. Die Abgrenzung zwischen den ideologischen Versatzstücken „Freiwilligkeit“, „Selbstlauftheorie“ und „Überspitzungen“" wurde im Herbst 1957 wieder fließend177: Der im Frühjahr 1959 selbst angegriffene Mückenberger zieh nun alle seit 1953 agrarpolitisch Andersdenkenden als Anhänger der „Selbstlauftheorie“; Fred Oelßner, 1957 wegen "Revisionismus" angefeindet, hatte seinerseits im August 1952 die herbeizitierten SED-Kreissekretäre für Landwirtschaft mit dem Vorwurf konfrontiert, die „Freiwilligkeit" der LPG-Gründung mit Untätigkeit zu verwechseln; Gerhard Grüneberg, der Mückenberger ablöste, hatte sich als 1. Sekretär des Bezirkes Frankfurt/ Oder 1957 durch ein überaus rigoroses Vorgehen im Herbeiführen von LPGGründungen profiliert, die ihm dann den Vorwurf der „Überspitzung“ eingebracht hatten. Offensichtlich rechnete die DBD es im Juli 1957 noch zu ihren Aufgaben, die „überspitzte“ Ausübung von Druck zu verhindern. Denn dem Bezirksvorsitzenden in Frankfurt/Oder, Pech, hielt das Präsidium vor, dagegen nichts unternommen zu haben ${ }^{178}$.

Die DBD sollte den Mißmut der ländlichen Bevölkerung über die Ausdehnung des sozialistischen Teils der Landwirtschaft und die Praxis, womit diese herbeigeführt wurde, dämpfen. Dazu entwickelte sie Argumentationen, die den Einzelbauern vermitteln sollten, daß die Kollektivierung nicht unter $\mathrm{Z}$ wang, sondern nach dem Willen der Bauern verlaufe ${ }^{179}$. Tatsächlich waren zumindest Teile der DBD-Funktionäre sehr genau darüber im Bilde, daß die MTS den Sozialismus nach ausgearbeiteten Plänen vorantrieben ${ }^{180}$. Das Präsidium der DBD setzte auf eine Arbeitsteilung mit der SED und gab eine Linie aus, derzufolge eine weitere Bildung nur in „geduldiger Überzeugungsarbeit und durch Erfahrungsaustausch von Einzel- und Genossenschaftsbauern " erfolgen dürfe.

Die 33. ZK-Tagung im Oktober 1957 beschloß ein Aktionsprogramm, das den Anteil des sozialistischen Teils der Landwirtschaft bis 1960 auf 50\% erhöhen sollte ${ }^{181}$. Das Sekretariat der DBD stimmte all dem zu ${ }^{182}$. Es war für die DBD bedeutsam, daß diese Tagung offiziell eine Agrarpolitik beendete, die noch auf Produktionssteigerung sowohl in der sozialistischen wie auch in der privaten Landwirtschaft gesetzt hatte, und statt dessen nun eine Erhöhung allein von sozialistischen Betrieben erwartete und förderte. Daneben diente die Tagung jedoch vor allem dazu, der SED wieder die politische Initiative auf dem Land zu sichern, über

176 SAPMO DY 30 IV 2/15/2, Mückenberger an Köhler, 14. 8. 1957; ebd., AG BO an Ulbricht, 8. 8. 1957.

177 SAPMO DY 30 IV 2/7/75, Protokoll ZK-Abt. LW, 26. 1. 1959.

178 ACDP VI-052-0320, Protokoll Präsidium, 23. 7. 1957.

179 Siehe dazu ACDP VI-052-244/2, Ausarbeitung Abt. Agrarpolitik der DBD 1957, „Beispiele für Überspitzungen, Verstöße gegen das Prinzip der Freiwilligkeit beim Eintritt in die LPG“, undatiert [2. Jahreshälfte 1957].

180 So hatte Pech für Frankfurt/Oder berichtet, daß bei den MTS-Bereichen eine genaue Planung mit Terminen und Orten zur Bildung von LPG vorliege; ACDP VI-052-0320, Protokoll Präsidium, 23. 7. 1957.

181 Schulz, „Kapitalistische Länder überflügeln“, S. 20f. Trotz Tatsachenverdrehung brauchbar: Noziczka, Politik, S. 49, 56; Nehrig/Noziczka, Weiterentwicklung.

182 ACDP VI-052-0535, Protokoll Sekretariat, 18.11. 1957, Beschlußvorlage für 3. PV-Sitzung. 
die sie in den Augen der ZK-Abteilung Landwirtschaft Mitte 1957 nicht verfügte ${ }^{183}$.

Die ehrgeizigen Pläne Ulbrichts, die sogar die Vorgaben der Plankommission übertrafen ${ }^{184}$, riefen in der ZK-Abteilung Landwirtschaft auch Diskussionen hervor. Die Abteilung stellte als Folge solch überspannter Zielformulierungen ein Vorauseilen einzelner Bezirke fest, die sich noch höhere Sozialisierungsziele setzen würden ${ }^{185}$. „Überspitzungen“ durch übergroßes Engagement und eine Radikalisierung in der Peripherie, wie im Bezirk Frankfurt/Oder anzutreffen, waren von der SED geduldet und durchaus erwünscht; es gibt jedenfalls keine Anzeichen dafür, daß diese unterbunden wurden, und die Karriere Grünebergs haben sie zweifellos befördert. Das Politbüro beschloß im Dezember 1957, daß die Leitungen der MTS - nach sozialistischer Diktion - gemeinsam mit den Mitgliedern der LPG, den Gemeindevertretungen und der VdgB den Plan zur sozialistischen Umgestaltung des Dorfes ausarbeiten sollten ${ }^{186}$.

Die DBD versuchte jeden Winkelzug der SED mitzumachen und paßte sich aufgrund der unmittelbaren Instruierung deren Kurs sogar schon an, noch bevor ihn die SED öffentlich verkündet hatte: Sie propagierte, die „Ständigen Arbeitsgemeinschaften " in LPG zu überführen und bestrafte nun ihre Mitglieder dafür, wenn diese, nur um einem LPG-Beitritt auszuweichen, solche „Arbeitsgemeinschaften" gründeten ${ }^{187}$. Bei Parteigremienwahlen weitete sie den Mandatsanteil der Genossenschaftsbauern zum Nachteil der Einzelbauern künstlich aus. Zwar wurden einzelbäuerliche Vertreter noch geduldet - immerhin stellten diese Mitte 1957 mit rund 55\% gegenüber gut 12\% LPG-Bauern das Gros unter den Anhängern ${ }^{188}$-, doch gerieten sie zusehends ins Hintertreffen. In den Wahlbestimmungen für die Delegiertenwahlen wurden sie nicht mehr gleichberechtigt mit den Genossenschaftsbauern genannt. Der im sozialistisch-genossenschaftlichen Segment der Agrarwirtschaft tätige Mitgliederanteil wurde mit allen Mitteln in den Vordergrund geschoben, genauso wie es die Arbeitsgruppe $\mathrm{BO}$ vorgegeben hatte. Diese ordnete nach der 33. ZK-Tagung an, die politische Auseinandersetzung in der Partei zu fördern - also Konflikte und Debatten zwischen den Funktionären und den einfachen Mitgliedern zu schüren, um die Basis daran zu schulen. Damit sollte der „Zustand überwunden [werden], daß sich ganze O[rtsgruppen] dieser uns am nächsten stehenden Partei gegen den Sozialismus aussprechen!“189

183 SAPMO DY 30 IV 2/7/75, Protokoll ZK-Abt. LW, 22. 7. 1957.

184 So Schulz, „Kapitalistische Länder überflügeln“, S. 20.

185 SAPMO DY 30 IV 2/7/79, Bl. 162 ff., Protokoll Abteilungsbesprechung, 16. 12. 1957.

186 Graffunder, Dialektik, S. 125. Zur Verantwortung der MTS gegenüber den Arbeitsgemeinschaften vgl. auch International Department, Rolle 109 (RGANI, 5-26-427, BI. 178187), Puschkin an ZK der KPdSU, 10. 10. 1956, Bericht über die Entwicklung einfachster Formen der Kooperativen in der Landwirtschaft der DDR (erstellt von Kulikow).

187 ACDP VI-052-0321, Protokolle Präsidium, 23. 10. 1957, 21. 11. 1957. Zur Forcierung der Werbung für LPG: ebd., Protokoll Präsidium, 16. 12. 1957.

188 Siehe Tabelle 7 und ACDP VI-052-245/2, Übersicht Anteil bäuerlicher Kollegen in LPG [1953-1958].

189 Zitat der AG BO in: SAPMO DY 30 IV 2/15/4, Dokument undatiert, nach 33. ZK-Plenum der SED. ACDP VI-052-0535, Protokoll Sekretariat, 4. 11. 1957, Vorlage 1. 11. 1957. ACDP VI-052-0321, Protokoll Präsidium, 21. 11. 1957. 